\title{
Duality in fuzzy linear programming: A survey
}

\author{
Guido Schryen · Diana Hristova
}

Received: date / Accepted: date

\begin{abstract}
The concepts of both duality and fuzzy uncertainty in linear programming have been theoretically analyzed, comprehensively and practically applied in an abundance of cases. Consequently, their joint application is highly appealing for both scholars and practitioners. However, the literature contributions on duality in fuzzy linear programming (FLP) are neither complete nor consistent. For example, there are no consistent concepts of weak duality and strong duality. The contributions of this survey are (1) to provide the first comprehensive overview of literature results on duality in FLP, (2) to analyze these results in terms of research gaps in FLP duality theory, and (3) to show avenues for further research. We systematically analyze duality in fuzzy linear programming along potential fuzzifications of linear programs (fuzzy classes) and along fuzzy order operators. Our results show that research on FLP duality is fragmented along both dimensions, more specifically duality approaches and related results vary in terms of homogeneity, completeness, consistency with crisp duality, and complexity. Fuzzy linear programming is still far away from a unifying theory as we know it from crisp linear programming. We suggest fur-
\end{abstract}

\author{
Guido Schryen \\ Department of Management Information Systems \\ University of Regensburg \\ Universitätsstraße 31 \\ 93053 Regensburg \\ Germany \\ Tel.: +49-941-9435634 \\ Fax: +49-941-9435635 \\ E-mail: guido.schryen@wiwi.uni-regensburg.de \\ Diana Hristova \\ Department of Management Information Systems \\ University of Regensburg \\ Universitätsstraße 31 \\ 93053 Regensburg \\ Germany \\ Tel.: +49-941-9436104 \\ Fax: +49-941-9436120 \\ E-mail: diana.hristova@wiwi.uni-regensburg.de
}


ther research directions, including the suggestion of comprehensive duality theories for specific fuzzy classes while dispensing with restrictive mathematical assumptions, the development of consistent duality theories for specific fuzzy order operators, and the proposition of a unifying fuzzy duality theory.

Keywords Fuzzy linear programming, Fuzzy decision making, Fuzzy duality, Duality theory, Weak duality, Strong duality, Complementary slackness condition, Fundamental theorem of duality

\section{Introduction}

Linear Programming (LP) is one of the most frequently applied OR techniques in real-world problems [64]. Traditional LP requires the decision maker to have deterministic and precise data available but this assumption is not realistic in many cases for several reasons [94, 2]: a) Many real life problems and models contain linguistic and/or vague variables and constraints. b) Collecting precise data is often challenging because the environment of the system is unstable or collecting precise data results in high information cost. c) Decision makers might not be able to express goals or constraints precisely because their utility functions are not defined precisely, or phenomena of the decision problem might only be described in a "fuzzy" way. Thus, being able to deal with vague and imprecise data may greatly contribute to the diffusion and application of LP. It should be noticed that the mentioned imprecision is not rooted in randomness but often in subjectivity of preferences and assessments. As a consequence, the use of probability distributions has not proved very successful in solving problems that show this type of uncertainty. Since Zadeh [92] introduced "Fuzzy sets", there exists a useful way of modeling vagueness in real life systems without having recourse to stochastic concepts.

Based on fuzzy sets, fuzzy decision making [4] and fuzzy mathematical programming, in particular Fuzzy Linear Programming (FLP), have been developed to tackle problems encountered in many different real-world applications [2, 64]. For example, FLP is used to solve problems in agricultural economics [55, 38, 16, 41, 48, 71, 70], network location [17], banking and finance [53, 54, 38, 27, 84, 37, 40], environment management $[72,52,42]$, inventory management $[49,66,65]$, manufacturing and production $[78,76,80,59,30,75,81,82,32,56]$, resource allocation $[1,36]$, supply chain management $[14,57,58,6,85]$, personnel management [73], media selection [83], trade balance [9], management of the reservoir watershed [12], dimension design [13], transportation management [78, 94, 10, 11, 22, 43, 68, 69, 79], product mix [33] and marketing [74].

The power of (crisp) LP includes valuable insights that are based on duality theory (see, for example, $[29,5]$ ). The particular usefulness of duality theory is not only given through its algorithmic (e.g., dual simplex algorithm) and mathematical benefits (e.g., weak/strong duality theorems), but it also includes explanatory power in economic interpretation [29, p. 203ff]. Together with the benefits of fuzzy mathematical programming, the joint application of fuzzy linear programming and duality theory is highly appealing for both scholars and practitioners but literature contributions on duality in FLP are neither complete nor consistent. For example, there are no 
consistent concepts of weak duality and strong duality. Striving for a comprehensive and consistent understanding of duality in FLP is an essential step towards jointly exploiting the power of duality and FLP, which are usually applied only separately.

The contributions of this survey are (1) to provide the first comprehensive overview of literature results on duality in FLP, (2) to analyze these results in terms of research gaps in FLP duality theory, and (3) to show avenues for further research. We systematically analyze duality in fuzzy linear programming along potential fuzzifications of linear programs and along fuzzy order operators.

The remainder of this paper is structured as follows: Section 2 presents the theoretical background regarding crisp linear duality theory, fuzzy sets and fuzzy arithmetic. In Section 3, we present the literature findings on duality in FLP. Section 4 analyzes our findings, identifies research gaps and suggests paths for further research, before Section 5 concludes the paper.

\section{Theoretical background}

In this section, we provide a brief overview of duality theory in linear programming, including key theorems and economic interpretations. We also provide a concise overview of fuzzy sets and fuzzy arithmetic.

\subsection{A brief overview of duality theory}

Linear programming and duality theory belong to the best understood fields in mathematical programming. We briefly present the most important findings of duality in linear programming (see, for example, $[29,5]$ ), and we later draw on these findings to assess the level of maturity of research on duality in fuzzy linear programming.

Given the primal (linear) problem ${ }^{1}$

$$
\begin{array}{ll}
\max & Z=c^{T} x \\
\text { subject to } & A x \leq b \\
& x \geq 0 \\
& c, x \in \mathbb{R}^{n} \\
& b \in \mathbb{R}^{m} \\
& A \in \mathbb{R}^{m x n},
\end{array}
$$

the dual problem ${ }^{2}$ is defined by

$$
\begin{array}{ll}
\min & W=b^{T} y \\
\text { subject to } & A^{T} y \geq c \\
& y \geq 0 .
\end{array}
$$

From a structural perspective, the dual problem is derived by a) transforming a maximization problem into a minimization problem, b) making the dimension of

\footnotetext{
1 Note that each linear problem can be easily transformed into the form given below.

2 Here, we define the dual problem by means of mathematical properties. An alternative, economic interpretation is given by Hillier and Lieberman [29, p. 203ff].
} 
the solution space (number of decision variables) the number of constraints and vice versa, c) substituting $\leq$ constraints with $\geq$ constraints, and d) switching the coefficients in the objective function with the right hand side values. We can also formulate more sloppy that variables become constraints and vice versa.

The key (structural) primal-dual relationships are as follows (proofs are included in many OR textbooks, including [29]):

Weak duality theorem: If $x$ is a feasible solution for the primal problem and $y$ is a feasible solution for the dual problem, then $c^{T} x \leq b^{T} y$.

Strong duality theorem: If $x^{*}$ is an optimal solution for the primal problem and $y^{*}$ is an optimal solution for the dual problem, then $c^{T} x^{*}=b^{T} y^{*}$.

Complementary slackness condition: Reformulating the functional constraints in the primal problem (1) and in the dual problem (2) by introducing "slack" variables, we yield $A x+x_{s}=b$ and $A^{T} y-y_{s}=c$, respectively. Let $\left(x, x_{s}\right)$ and $\left(y, y_{s}\right)$ be feasible solutions of the primal and the dual problem, respectively. Then, $\left(x, x_{s}\right)$ and $\left(y, y_{s}\right)$ are optimal solutions of the problems if and only if $x^{T} y_{s}+x_{s}^{T} y=0$.

Fundamental theorem of duality: The following relationships are the only possible ones between the primal and the dual problems:

1. If one problem has feasible solutions and a bounded objective function (and so an optimal solution), then so does the other problem.

2. If one problems has feasible solutions and an unbounded objective function (and so no optimal solution), then the other problem has no feasible solutions.

3. If one problem has no feasible solutions, then the other problem has either no feasible solutions or an unbounded objective function.

Due to the strong duality theorem, it is sufficient to solve the dual problem in order to get the optimal solution value of the primal problem. This property can also be proved when we draw on Lagrangian relaxation. For example, Gordon [23] uses the Lagrangian multiplier theorem to show that the problem $\left(\min c^{t} x\right.$ s.t. $\left.A x \leq b\right)$ has the same optimal solution as the problem ( $\max -b^{t} y$ s.t. $\left.A^{t} y=-c^{t}, y \geq 0\right)$, assumed that both problems have bounded optimal solutions. We can easily apply this result on our primal-dual problems by transforming the non-negativity constraints $(x \geq 0)$ into $(-x \leq 0)$ and using the transformation $(\max f(x)=-\min -f(x))$. Rödder and Zimmermann [63] use an economic interpretation ${ }^{3}$ of the Lagrangian function for the primal problem (1) and show that the saddle point $\left(x^{*}, y^{*}\right)$ of the Lagrangian function represents the optimal solutions $x^{*}$ and $y^{*}$ of the primal and the dual problem, respectively.

The usefulness of duality theory is manifold. First, it is of algorithmic value as (1) it is sufficient to solve the easier problem of the primal-dual problem pair in order to obtain the optimal solutions for both problems, (2) if the primal problem has an optimal solution, then the objective value of any feasible solution of the dual problem is an upper bound of the optimal objective value of the primal problem (due to the

3 The researchers interpret $x$ as production units and $y$ as resource prices on the market. 
weak duality theorem), and (3) it is the root of the dual Simplex algorithm. A second major benefit of duality theory lies in its economic interpretation: in economic contexts, the objective function often corresponds to profit and the functional constraints often represent constraints of resources, such as machine hours or financial budgets. For each basic feasible solution of the primal problem, the corresponding objective value of the dual problem is given by $W=\sum_{i=1}^{m} b_{i} y_{i}$. Consequently, each $b_{i} y_{i}$ can be interpreted as the contribution to profit by having $b_{i}$ units of resource $i$ available for the primal problem. In other words, the dual variable $y_{i}$ can be interpreted as the contribution to profit per unit of resource $i$, when the current set of basic variables is used to obtain the primal solution. This property is particularly useful for the optimal basic solution in the primal problem because each $y_{i}^{*}$ value in the optimal solution in the dual problem gives the marginal increase of profit if the corresponding resource is increased by 1 ("shadow prices"). Also the complementary slackness condition can be interpreted in this economic context: if a constraint in the primal problem is not binding (the corresponding slack variable is positive), then the shadow price must equal zero. If the shadow price is positive, then the corresponding constraint must be binding (the slack variable equals zero). This holds for the dual problem, too, due to the symmetry property (the dual problem of the dual problem is the primal problem). For a more comprehensive interpretation of the economic meaning of the dual problem see [29, p. 203ff].

\subsection{A concise overview of fuzzy sets and fuzzy arithmetic}

Fuzzy set theory goes back to Zadeh [92], who proposed fuzzy sets as means for dealing with non-probabilistic uncertainty. The key idea of fuzzy set theory is the extension of the (crisp) membership concept in traditional set theory by providing for a degree with which an element belongs to a set. The degrees are specified by a membership function.

Among the many introductory books on fuzzy sets and fuzzy optimization, we mainly draw on references $[96,97,19,64]$ to briefly present the very basics. We present only those subfields of fuzzy set theory and fuzzy arithmetic that are relevant for (duality theory in) FLP.

Definition 1 (Fuzzy set) Let $X$ be a crisp set. Then we define a fuzzy set $A$ in $X$ as a set of ordered pairs

$$
A:=\left\{\left(x, \mu_{A}(x)\right) \mid x \in X\right\} .
$$

$\mu_{A}: X \rightarrow \mathbb{R}$ is called the membership function. Important concepts of fuzzy sets are:

- Normality: If $\sup _{x} \mu_{A}(x)=1$, then the fuzzy set $A$ is called normal.

- Support: The support (0-level set) of a fuzzy set $A$ is the set $S(A)=\left\{x \in X \mid \mu_{A}(x)>0\right\}$.

- Core: The core of a fuzzy set $A$ is the set $C(A)=\left\{x \in X \mid \mu_{A}(x)=1\right\}$.

- $\alpha$-cuts: The $\alpha$-level set (or $\alpha$-cut) $A[\alpha]$ of a fuzzy set $A$ in $X$ is defined by $A[\alpha]=$ $\left\{x \in X \mid \mu_{A}(x) \geq \alpha\right\}, 0<\alpha \leq 1$. For $X=\mathbb{R}$, the 0 -level set $A[\alpha]$ is defined as the closure of the set $\left\{x \in \mathbb{R} \mid \mu_{A}(x)>0\right\}$. 
- Convexity: A fuzzy set $A$ is convex if

$$
\mu_{A}\left(\lambda x_{1}+(1-\lambda) x_{2}\right) \geq \min \left\{\mu_{A}\left(x_{1}\right\}, \mu_{A}\left(x_{2}\right)\right\}, x_{1}, x_{2} \in X, \lambda \in[0 ; 1]
$$

i.e. if its membership function is quasi-concave.

Apparently, a key difference between crisp and fuzzy sets is their membership function; a crisp set is conceptually a specific fuzzy set with the membership function $\mu_{A}(x)=1$, if $x \in A, \mu_{A}(x)=0$ else.

While set-theoretic operations on crisp fuzzy sets are defined consistently, various implementations of set-theoretic operations on fuzzy sets have been proposed: For example, Zadeh [92] defines the intersection $A \cap B$ and the union $A \cup B$ of two fuzzy sets $A$ and $B$ in $X$ as

$$
A \cap B=\left\{\left(x, \mu_{A \cap B}(x)\right)\right\}, \mu_{A \cap B}(x)=\min \left\{\mu_{A}(x), \mu_{B}(x)\right\}
$$

and

$$
A \cup B=\left\{\left(x, \mu_{A \cup B}(x)\right)\right\}, \mu_{A \cup B}(x)=\max \left\{\mu_{A}(x), \mu_{B}(x)\right\},
$$

respectively. As the max and the min operators usually do not correspond to what is meant by "and/intersection" and "or/union", other operator implementations have been proposed, including the Hamacher operators, the Yager operators and the Dubois and Prade operators [97, p. 29ff].

A general class of intersection/union operators for fuzzy sets has been defined as t-norms/t-conorms (s-norms) [19, 20, p. 17, p. 90].

Definition 2 (t-norm/t-conorm) t-norms (t-conorm) are two-valued functions $t$ that map from $[0,1] \times[0,1]$ into $[0,1]$ and that satisfy the following conditions:

1. $t(0,0)=0 ; t\left(\mu_{A}(x), 1\right)=t\left(1, \mu_{A}(x)\right)=\mu_{A}(x), x \in X$ (t-norm)

$$
t(1,1)=1 ; t\left(\mu_{A}(x), 0\right)=t\left(0, \mu_{A}(x)\right)=\mu_{A}(x), x \in X \text { (t-conorm) }
$$

2. $t\left(\mu_{A}(x), \mu_{B}(x)\right) \leq t\left(\mu_{C}(x), \mu_{D}(x)\right)$

if $\mu_{A}(x) \leq \mu_{C}(x)$ and $\mu_{B}(x) \leq \mu_{D}(x)$ (monotonicity)

3. $t\left(\mu_{A}(x), \mu_{B}(x)\right)=t\left(\mu_{B}(x), \mu_{A}(x)\right)$ (commutativity)

4. $t\left(\mu_{A}(x), t\left(\mu_{B}(x), \mu_{C}(x)\right)\right)=t\left(t\left(\mu_{A}(x), t\left(\mu_{B}(x)\right), \mu_{C}(x)\right)\right.$ (associativity)

In the field of fuzzy optimization, a particular type of fuzzy sets, fuzzy numbers $\mathbb{F}(\mathbb{R})$, are used. They are defined by Dubois and Prade [19] as a special case of a fuzzy interval.

Definition 3 (Fuzzy interval and fuzzy number) A fuzzy interval $\tilde{y}$ is a fuzzy set in $X=\mathbb{R}$ whose membership function $\mu_{\tilde{y}}(c \leq a \leq b \leq d, a, b, c, d \in \mathbb{R})^{4}$ is

1. a continuous mapping from $\mathbb{R}$ to $[0,1]$,

2. constant on $(-\infty, c]: \mu_{\tilde{y}}(x)=0 \forall x \in[-\infty, c]$,

3. strictly increasing on $[c, a]$,

4. constant on $[a, b]: \mu_{\tilde{y}}(x)=1 \forall x \in[a, b]$,

5. strictly decreasing on $[b, d]$,

\footnotetext{
${ }^{4}$ Eventually, we can also have $c=-\infty$, or $a=b$, or $c=a$, or $b=d$, or $d=\infty$.
} 
6. constant on $[d, \infty]: \mu_{\tilde{y}}(x)=0 \forall x \in[d, \infty]$.

A fuzzy number is the special case of a fuzzy interval, where $a=b$.

Unfortunately, there is some terminological confusion in the literature regarding the definition of fuzzy numbers as many authors, e.g., [7, 15, 18, 34, 46, 50] allow in their definitions of fuzzy numbers a core of more than one element and refer to Dubois and Prade [19] fuzzy intervals as fuzzy numbers, which is not in accordance with Definition 3. In order to remain consistent with this widely used terminology and to avoid confusion, we adopt this (broader) understanding of fuzzy numbers in the remainder of this paper. This approach is consistent with Zimmermann [97]. ${ }^{5} \mathrm{We}$ also mark in Table 1 those papers which use an understanding different from Dubois and Prade [19].

Definition 4 (Spread) The left spread of a fuzzy number $\widetilde{y}$ with a membership function $\mu_{\tilde{y}}(c \leq a \leq b \leq d, a, b, c, d \in \mathbb{R})$ is defined as $(a-c)$. The right spread of $\tilde{y}$ is defined as $(d-b)$.

Definition 5 (Specific types of fuzzy numbers) A fuzzy number $y=(c, a, b, d)$ with membership function $\mu_{\tilde{y}}$ is called

- trapezoidal if $\mu_{\tilde{y}}$ is piecewise linear and $a<b$,

- triangular if $\mu_{\tilde{y}}$ is piecewise linear and $a=b$,

- symmetric if $\mu_{\tilde{y}}(a-h)=\mu_{\tilde{y}}(b+h) \forall h \geq 0$,

- an LR type fuzzy number if

$$
\mu_{\tilde{y}}(x)=\left\{\begin{array}{l}
L\left(\frac{m-x}{\alpha}\right) \text { for } x \leq m, \alpha>0 \\
R\left(\frac{x-m}{\beta}\right) \text { for } x \geq m, \beta>0
\end{array}\right.
$$

with $L(x)=L(-x), L(0)=1, R(x)=R(-x), R(0)=1, L, R$ continuous and nonincreasing on $[0, \infty)$.

Let $X$ be a nonempty subset of $\mathbb{R}$. The membership function $\mu_{\tilde{y}}$ is called (see, for example, [61])

- quasi-concave on $X$ if

$$
\mu_{\widetilde{y}}(\lambda x+(1-\lambda) y) \geq \min \left\{\mu_{\tilde{y}}(x), \mu_{\tilde{y}}(y)\right\}
$$

for every $x, y \in X$ and every $\lambda \in(0,1)$ with $\lambda x+(1-\lambda) y \in X$,

- strictly quasi-concave on $X$ if

$$
\mu_{\tilde{y}}(\lambda x+(1-\lambda) y)>\min \left\{\mu_{\widetilde{y}}(x), \mu_{\widetilde{y}}(y)\right\}
$$

for every $x, y \in X, x \neq y$, and every $\lambda \in(0,1)$ with $\lambda x+(1-\lambda) y \in X$,

5 "Nowadays, definition 5-3 [ defining a fuzzy number with a core of one element] is very often modified. For the sake of computational efficiency and ease of data acquisition, trapezoidal membership functions are often used. [...] Strictly speaking, it [the fuzzy set with trapezoidal membership functions] is a fuzzy interval $[\ldots] "$ ( . p. 59) 
Fig. 1 Sum and product of fuzzy numbers $\widetilde{3}$ and $\widetilde{6}$

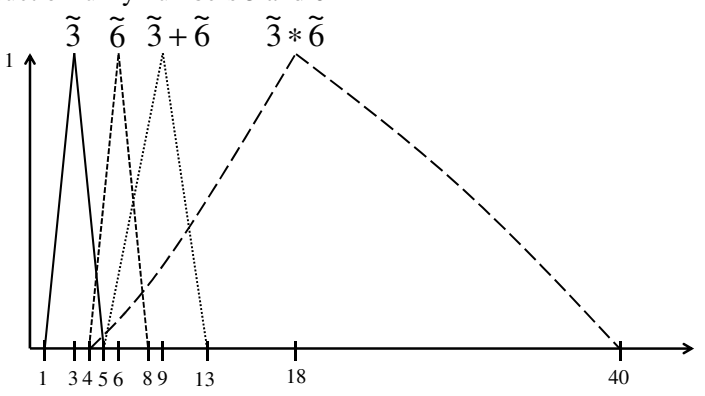

Note: $\tilde{3} * \tilde{6}$ is not a triangular fuzzy number.

- semistrictly quasi-concave on $X$ if $\mu_{\tilde{y}}$ is quasi-concave on $X$ and (4) holds for every $x, y \in X$ and every $\lambda \in(0,1)$ with $\lambda x+(1-\lambda) y \in X, \mu_{\widehat{y}}(\lambda x+(1-\lambda) y) \in$ $(0,1)$ and $\mu_{\tilde{y}}(x) \neq \mu_{\tilde{y}}(y)$.

Fuzzy arithmetic operations are defined by Dubois and Prade [18] based on the extension principle proposed by Zadeh [89, 90, 91]:

Definition 6 (Fuzzy arithmetic) Let $\widetilde{a}$ and $\widetilde{b}$ be two fuzzy numbers with membership functions $\mu_{\tilde{a}}$ and $\mu_{\tilde{b}}$. The membership function $\mu_{\tilde{c}}$ of the fuzzy number that results from the operation $\widetilde{c}=\widetilde{a} \circ \widetilde{b}, \circ \in\{+,-, *, /\}$, is defined as

$$
\mu_{\widetilde{c}}(z)=\sup _{x \circ y=z} \min \left\{\mu_{\widetilde{a}}(x), \mu_{\widetilde{b}}(y)\right\} .
$$

An equivalent definition draws on $\alpha$-cuts and interval arithmetic [35, chapter 4]. Figure 1 demonstrates arithmetic on triangular fuzzy numbers in a sample case.

While there is a consensus in the literature on how to perform arithmetic operations on fuzzy numbers, there is no universally accepted way to order fuzzy numbers. A key concept of ordering fuzzy numbers is the usage of a ranking function $R: \mathbb{F}(\mathbb{R}) \rightarrow \mathbb{R}$, with $\widetilde{a} \preceq \widetilde{b}$ if and only if $R(\widetilde{a}) \leq R(\widetilde{b})$ and $\widetilde{a} \approx \widetilde{b}$ if and only if $R(\widetilde{a})=R(\widetilde{b})$ (see, for example, [50]). $R$ is called linear if and only if $R(\widetilde{a}+\lambda \widetilde{b})=$ $R(\widetilde{a})+\lambda R(\widetilde{b}), \lambda \in \mathbb{R}$. A second option is to draw on a (lexicographic) ranking function $R^{\prime}: \mathbb{F}(\mathbb{R}) \rightarrow \mathbb{R} \times \mathbb{R}[28]$ based on the concepts of possibilistic mean value and variance of a fuzzy number [8]. Third, ranking fuzzy numbers can also draw on indices based on possibility theory [21].

While ranking functions support a crisp decision of whether a fuzzy number $\widetilde{a}$ is smaller than or equal to a fuzzy number $\widetilde{b}$, a different approach based on fuzzy relations assigns a value with which $\widetilde{a}$ is smaller than or equal to $\widetilde{b}$ [61]. The relation $\preceq$ on $F(\mathbb{R}) \times F(\mathbb{R})$ is valued such that $\mu_{\preceq}: F(\mathbb{R}) \times F(\mathbb{R}) \rightarrow[0,1]$. $\mu_{\preceq}(\widetilde{a}, \widetilde{b})$ can be regarded as the degree with which $\widetilde{a}$ is smaller than or equal to $\widetilde{b}$.

While fuzzy relations, which enable the comparison of fuzzy numbers, are applied to fuzzy subsets of $F(\mathbb{R}) \times F(\mathbb{R})$, Zimmermann [95] proposes an extension of comparison operators of crisp numbers in order to compare crisp numbers in a fuzzy 
sense. Here, relations are fuzzy subsets of $\mathbb{R} \times \mathbb{R}$, i.e. the relation $\preceq$ on $\mathbb{R} \times \mathbb{R}$ is valued such that $\mu_{\preceq}: \mathbb{R} \times \mathbb{R} \rightarrow[0,1]$.

In the literature a fuzzification of the max and min operations has been proposed. One of the key concepts was proposed by Dubois and Prade [18]:

Definition 7 (Fuzzy max/min) Let $\widetilde{a}$ and $\widetilde{b}$ be two fuzzy numbers with membership functions $\mu_{\tilde{a}}$ and $\mu_{\tilde{b}}$. The "fuzzy max" of $\widetilde{a}$ and $\widetilde{b}$, which we denote by $\widetilde{c}=\widetilde{\max }(\widetilde{a}, \widetilde{b})$, has a membership function $\mu_{\tilde{c}}$ such that

$$
\mu_{\widetilde{c}}(z)=\max _{\max \{x, y\}=z} \min \left\{\mu_{\widetilde{a}}(x), \mu_{\widetilde{b}}(y)\right\} .
$$

In the same way, $\widetilde{d}=\widetilde{\min }(\widetilde{a}, \widetilde{b})$ has a membership function $\mu_{\widetilde{d}}$ such that

$$
\mu_{\widetilde{d}}(z)=\max _{\min \{x, y\}=z} \min \left\{\mu_{\widetilde{a}}(x), \mu_{\widetilde{b}}(y)\right\} .
$$

In the next section we present the results of our literature review.

\section{Duality in fuzzy linear programming}

In order to structure the findings of the literature, FLP models are classified according to the components that can be fuzzified. In a FLP, the maximization of the objective function $(\max )$, the order operator of constraints $(\leq)$, the limit values of constraints $(b)$, the coefficients of the objective function $(c)$, the coefficients of constraints $(A)$, and the decision variables can be fuzzified. Each of the model components can be either crisp or fuzzy. Because of the limited practical usefulness of fuzzy decision variables (cf. succeeding paragraph) and in order to keep the number of classes manageable, we do not explicitly provide a dimension for the type of decision variables.

When decision variables are fuzzy, the optimal solutions of such problems are also fuzzy. However, in most real-world applications decision makers require crisp solutions to make a decision. For example, Nasseri et al. [51] consider a FLP profit maximization problem, where the produced units of products are represented as fuzzy sets. This implies that the optimal number of units to be produced is also a fuzzy number. However, companies can produce only a crisp number of units, which needs to be considered in the setup of production machines. On the other hand, fuzzy solutions can have practical benefits in situations where crisp decisions are not derived directly from fuzzy solutions, but fuzzy solutions represent the input for further optimization problems. For example, fuzzy solutions might be the result of an optimization problem that looks for optimal market prices of a good. These fuzzy prices can then be used as (fuzzy) coefficients in a further optimization problem that looks for (crisp) solutions of a production plan. However, we still admit that the practical applicability of fuzzy solutions is limited. For the sake of completeness of theoretical approaches, we discuss LP models with fuzzy variables in Subsection 3.5.

Overall, our classification results in a number of $2^{5}-1=31$ potential classes of fuzzy linear programs if we require a model to have at least one fuzzy component. A similar classification is proposed by Baykasoglu and Goecken [2], but the researchers 
do not distinguish between fuzzifying the order operator and the limit values of the constraints. However, since a fuzzy order operator can also be used to compare crisp numbers (see section 2.2) and fuzzy numbers can only be compared by a fuzzy order operator, we consider this separation necessary.

Table 1 shows the resulting classes and the references that contribute to the duality theory of the respective class. While columns represent (five) model components, which can be fuzzified, rows correspond to (31) classes of fuzzy linear programs. The symbol $\checkmark$ in row no. $i$ and column no. $j$ indicates that class $i$ is fuzzified with regard to model component $j$; otherwise the table entry is empty. Table 1 shows that some of the 31 potential classes of fuzzy linear programs are impossible and are thus excluded from further consideration: a) When coefficients in the objective function are fuzzy then the objective value is fuzzy, too, and underlies a fuzzy maximization operator. Consequently, classes 2-3,6-7,10-11, and 14-15 are excluded. b) Constraint operators $(\leq)$ must be fuzzy if $b, A$, or both are fuzzy. Thus, classes $1,3,4-7,17$, and 19-23 are excluded. To sum up, among the 31 potential classes of fuzzy linear programs only 14 classes remain feasible. We found that among these 14 classes, only seven classes (classes 8,16,18,24,28,30,31) have been addressed in the literature. The last column lists references for each of these classes; blank entries indicate that we have not found any reference for the respective class.

Some of the existing papers work with arbitrary symmetric trapezoidal fuzzy numbers as a definition of the right-hand side of a constraint, which is not in accordance with the idea behind the constraints in FLP. In particular, they allow a fuzzy right-hand side to be modeled as a fuzzy number with a non-zero left/right spread, which implies that there does not exist a level under/over which the left-hand satisfies the constraint with certainty. This contradicts the interpretation of the constraints of FLP program, where a tolerance level above/below the certainty level is given. However, since most of the trapezoidal fuzzy numbers discussed in the presented works can be additionally restricted to have a zero left/right spread, we discuss the approaches further under the condition that they are only applicable with this additional restriction. An exception here is Nasseri et al. [51] where the authors require the use of fuzzy numbers with positive left and right spread. As a result, we believe that the paper does not provide a useful contribution to duality in FLP and exclude it from further discussion.

Table 1 shows that with regard to fuzzy models of class 28 we found two papers in the literature. These approaches use fuzzy decision variables in the primal problem and crisp decision variables in the dual problem. However, the authors do not provide a sound justification for simply mapping fuzzy numbers onto crisp numbers. We thus argue that the presented approaches do not provide any additional value to the field of fuzzy duality theory and thus exclude these from further discussions. As a result, the number of considered classes reduces to six. Finally, as discussed in the previous section, we mark those papers which define fuzzy numbers different from Definition 3.

We now present literature results for those feasible classes where we found references (classes 8,16,18,24,30,31). The order of presentation is in the order of ascending numbers of classes with two exceptions. The first one is due to the fact that many papers draw on the early ideas of Hamacher et al. [26] and Rödder and Zimmermann 
Table 1 Classification of fuzzy linear programs.

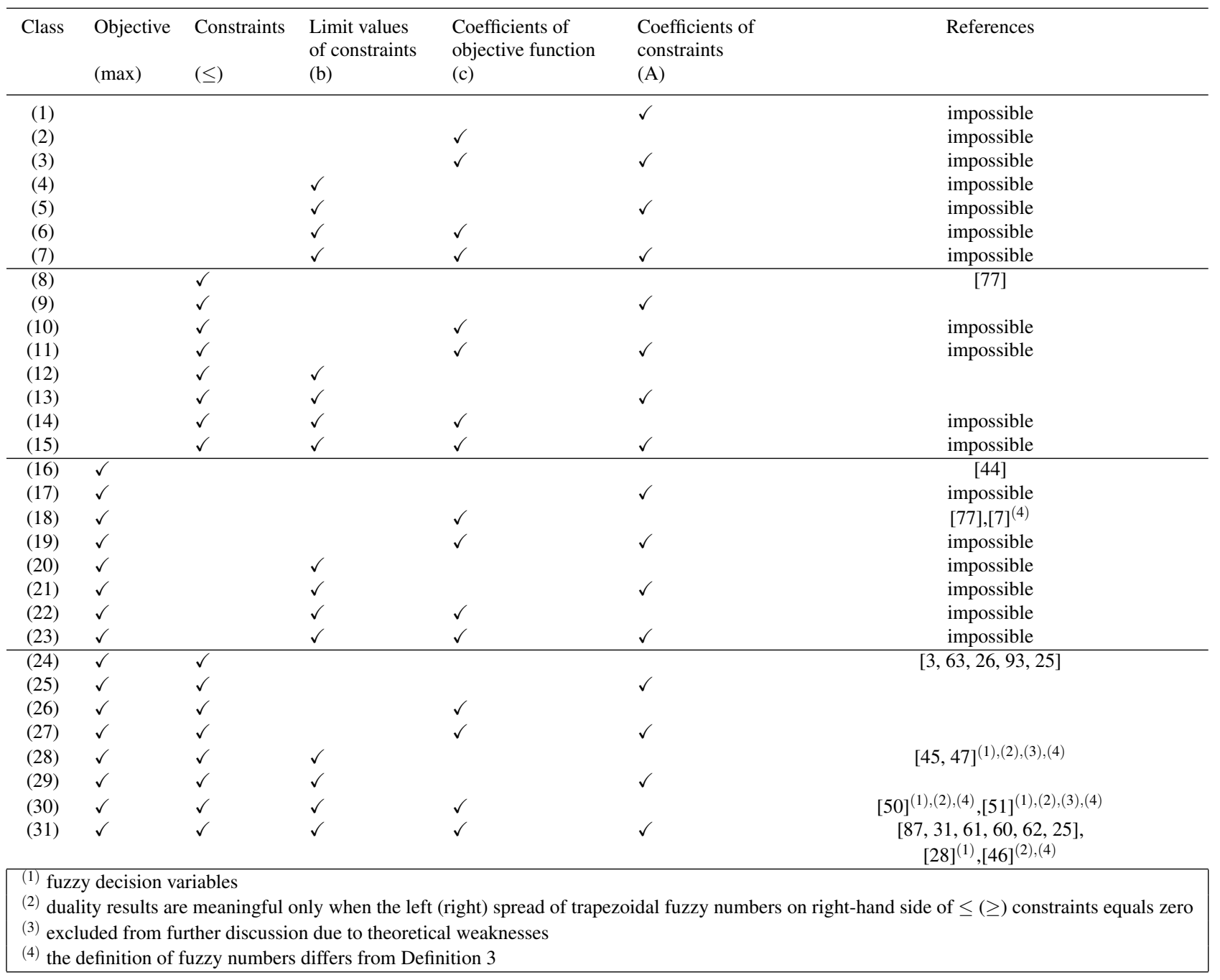

[63], which are used for deriving duality results in class 24 . Thus, this class is presented first. The second exception is due to the separate discussion in Subsection 3.5 of approaches, which consider fuzzy decision variables.

In labeling the optimization problems we generally used the following convention: primal problems in subsection $i$ are labeled $(P i)$; dual problems in subsection $i$ are labeled $(D i)$. In case of more than one approach in a given subsection and difference between the primal or dual problems, we use lower case letters to differentiate between the approaches, i.e. the first primal problem in subsection $i$ is labeled (Pia). Derived problems are labeled using additional prime marks. Important auxiliary prob- 
lems are labeled $(A i)$, where $i$ is initialized at one and increased with the number of labeled problems.

In the following subsections, we present the duality results of the literature regarding the various classes of fuzzy linear problems with a particular focus on duality theorems as they are known from duality theory of crisp linear programming. We want to note that (1) while we checked the literature for included proofs of duality theorems, we did not investigate whether further duality theorems can be derived from the findings of the respective researchers as we believe that such an investigation is beyond the scope of a literature review, and (2) some of the papers draw on formulations of "strong duality" and "complementary slackness" that are slightly different from the common definitions presented in Subsection 2.1.

\subsection{Class 24: Fuzzy maximization and fuzzy constraints}

The primal optimization problem of this class looks as follows:

$$
\begin{array}{ll}
\widetilde{\max _{x}} & c^{t} x \\
\text { s.t. } & A x \preceq b \\
& x \geq 0
\end{array}
$$

In this case, there is only uncertainty regarding the maximization and comparison

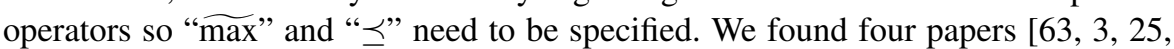
26 ] which deal with $(P 1)$ as its primal problem and present them in the following subsections. At the end of this section we provide a brief overview of the discussed approaches.

\subsubsection{Approach of Rödder and Zimmermann (1980)}

The paper by Rödder and Zimmermann [63] does not directly consider problem $(P 1)$ as its primal problem. However, as it deals with uncertainty regarding the optimal value of the objective as well as the degree of satisfaction of the constraints of the crisp equivalent of $(P 1)$, we present it here. Rödder and Zimmermann [63] define their approach based on the idea that the solution of $(P 1)$ in the crisp case is the one that maximizes the Lagrangian function:

$$
L(x, y)=\underbrace{c^{t} x}_{\text {primary }}+\underbrace{y^{t}(b-A x)}_{\text {secondary }} .
$$

The researchers interpret $L(x, y)$ as the total profit for a producer (primary plus secondary), who produces $x$, where $y$ represents the price of production resources on the market, $c$ contains the prices for the production on the market, $A$ is the technology matrix and $b$ are the available resource capacities of the producer.

In a fuzzy setting the producer does not maximize his profit, but rather has a certain aspiration level $c^{t} x^{0}$ for the primary profit that $\mathrm{s} / \mathrm{he}$ aims to reach, i.e. the maximization operator in the primal problem is fuzzy. Moreover, the producer is said to have a degree of satisfaction from his secondary profit $y^{t}(b-A x)$ as a function of the prices 
on the market $y$. Let the membership functions of the primary and the secondary profit be $\mu_{\succeq}\left(c^{t} x, c^{t} x^{0}\right)$ and $\mu_{\preceq}(A x, b, y)$, respectively. To solve $(P 1)$, the researchers follow the symmetrical approach of [4] so that in such an optimization problem the objective is treated as a constraint and the solution is the one that maximizes the intersection of the membership functions of the objective and the constraints. Thus, the solution of $(P 1)$ is, for given price of the resources on the market $y$, the one that satisfies

$$
x=\operatorname{argmax}_{x \geq 0}\left(\min \left\{\mu_{\succeq}\left(c^{t} x, c^{t} x^{0}\right), \mu_{\preceq}(A x, b, y)\right\}\right) .
$$

The primal problem $(P 1)$ can then be rewritten as

$$
\begin{array}{ll} 
& \max _{x, \alpha} \alpha \\
\text { s.t. } & \mu_{\succeq}\left(c^{t} x, c^{t} x^{0}\right) \geq \alpha \\
& \mu_{\preceq}(A x, b, y) \geq \alpha \\
& x \geq 0 \\
& \alpha \in \mathbb{R}
\end{array}
$$

Note that the primal problem is defined as a parametric problem for a given $y$ and its optimal solution is a pair $\left(\alpha(y), x^{*}(y)\right)$.

The dual problem is defined analogously to the primal problem, however, in this case the decision-maker is not the producer, but the market with an analogous interpretation. The membership functions of the objective of the dual problem and the constraints are $\mu_{\preceq}\left(b^{t} y, b^{t} y^{0}\right)$ and $\mu_{\succ}\left(A^{t} y, c, x\right)$ respectively. The dual problem can then be rewritten as a parametric problem of $x$ with an optimal solution $\left(\kappa(x), y^{*}(x)\right)$ :

$$
\begin{array}{ll} 
& \min _{y, \kappa} \kappa \\
\text { s.t. } & -\mu_{\preceq}\left(b^{t} y, b^{t} y^{0}\right) \leq \kappa \\
& -\mu_{\succeq}\left(A^{t} y, c, x\right) \leq \kappa \\
& y \geq 0 \\
& \kappa \in \mathbb{R}
\end{array}
$$

The researchers define:

$$
\begin{aligned}
& X^{0}=\left\{x \geq 0 \mid \forall y \geq 0: y^{t} b<0 \Rightarrow y^{t} A x \leq 0\right\} \\
& Y^{0}=\left\{y \geq 0 \mid \forall x \geq 0: c^{t} x>0 \Rightarrow y^{t} A x \geq 0\right\}
\end{aligned}
$$

as the set of solutions of $(P 1 a)$ and $(D 1 a)$ respectively, where the two problems are bounded. To prove duality, the researchers restrict the sets $X^{0}$ and $Y^{0}$ to the sets $X$ and $Y$ respectively:

$$
\begin{aligned}
& X=\left\{x \geq 0 \mid \forall y \geq 0: y^{t} b \leq 0 \Rightarrow y^{t} A x \leq 0\right\} \\
& Y=\left\{y \geq 0 \mid \forall x \geq 0: c^{t} x \geq 0 \Rightarrow y^{t} A x \geq 0\right\}
\end{aligned}
$$

and by applying Farkas' lemma show that they are equivalent to:

$$
\begin{aligned}
& X=\{x \geq 0 \mid \exists \beta \in \mathbb{R}, \beta \geq 0, \beta b \geq A x\} \\
& Y=\left\{y \geq 0 \mid \exists \gamma \in \mathbb{R}, \gamma \geq 0, \gamma c^{t} \leq y^{t} A\right\}
\end{aligned}
$$


The researchers then restrict the solution set of $(P 1 a)$ and $(D 1 a)$ to $X$ and $Y$ as follows:

$$
\begin{array}{ll} 
& \max _{x, \alpha, \beta} \alpha \\
\text { s.t. } & \mu_{\succeq}\left(c^{t} x, c^{t} x^{0}\right) \geq \alpha \\
& \mu_{\preceq}(A x, b, y) \geq \alpha \\
& x \geq 0 \\
& \alpha \in \mathbb{R} \\
& \beta b \geq A x \\
& \gamma c^{t} \leq y^{t} A \\
& \beta \geq 0 \\
& \gamma \geq 0 \\
& \min _{y, \kappa, \gamma} \kappa \\
\text { s.t. } & -\mu_{\preceq}\left(b^{t} y, b^{t} y^{0}\right) \leq \kappa \\
& -\mu_{\succeq}\left(A^{t} y, c, x\right) \leq \kappa \\
& y \geq 0 \\
& \kappa \in \mathbb{R} \\
& \beta b \geq A x \\
& \gamma c^{t} \leq y^{t} A \\
& \beta \geq 0 \\
& \gamma \geq 0
\end{array}
$$

Note that in $\left(P 1 a^{\prime}\right) \gamma$ and $y$ are given as parameters, while in $\left(D 1 a^{\prime}\right) \beta$ and $x$ are given as parameters.

Weak duality For any two optimal solutions $\left(\alpha(y), x^{*}(y), \beta(y)\right)$ and $\left(\kappa(x), y^{*}(x), \gamma(x)\right)$ of $\left(P 1 a^{\prime}\right)$ and $\left(D 1 a^{\prime}\right)$ respectively the following holds:

$$
\begin{aligned}
& \frac{\gamma}{\beta(y)} c^{t} x^{*}(y) \leq b^{t} y \quad \forall y \in Y \\
& c^{t} x \leq \frac{\beta}{\gamma(x)} b^{t} y^{*}(x) \quad \forall x \in X
\end{aligned}
$$

Note that the presented weak duality theorem is a relation of the elements of $X$ and $Y$ and would hold regardless of the formulation of $(P 1 a)$ and $(D 1 a)$ and regardless of whether $x$ and $y$ are optimal.

\subsubsection{Approach of Bector and Chandra (2002)}

The paper by Bector and Chandra [3] also addresses $(P 1)$ as the primal problem and modifies the approach by Rödder and Zimmermann [63]. To compare crisp numbers in a fuzzy sense, given two real numbers $A$ and $B, A \preceq B$ is defined in the paper using a linear, monotone membership function $\mu_{\preceq}: \mathbb{R} \times \mathbb{R} \rightarrow[0,1]$ as follows:

$$
\mu_{\preceq}(A, B)= \begin{cases}0 & A>B+f_{0} \\ 1-\frac{A-B}{f_{0}} & B<A \leq B+f_{0} \\ 1 & A \leq B\end{cases}
$$

where $f_{0}>0$ is the tolerance interval describing the extent to which the constraint can be violated. $\mu_{\succeq}$ is defined analogously. 


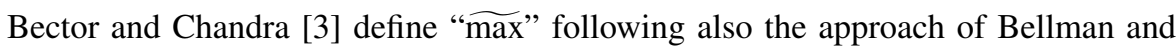
Zadeh [4]. The researchers transform the objective into a constraint using the aspiration level $c^{t} x^{0}$. (P1) can then be rewritten as:

$$
\begin{aligned}
& c^{t} x \succeq c^{t} x^{0} \\
& A x \preceq b \\
& x \geq 0 .
\end{aligned}
$$

The solution of $(P 1 b)$ is the pair $(x, \alpha)$ such that

$$
\begin{array}{ll} 
& \max _{x, \alpha} \alpha \\
\text { s.t. } & \mu_{\succeq}\left(c^{t} x, c^{t} x^{0}\right) \geq \alpha \\
& \mu_{\preceq}(A x, b) \geq \alpha \\
& x \geq 0 \\
& \alpha \in[0,1] .
\end{array}
$$

Note that $\mu_{\preceq}(A x, b)$ is not a single number, but rather a vector containing, for each constraint $i$, the value $\mu_{\preceq(i)}\left(A_{i} x, b_{i}\right)$, where $\mu_{\preceq(i)}$ stands for the corresponding membership function. Let the tolerance interval of the membership function $\mu_{\preceq(i)}$ be $p_{i}$ (see (8)) and $p=\left(p_{1}, \ldots, p_{m}\right)$. Let, in addition $p_{0}$ be the tolerance interval of the membership function $\mu_{\succeq}\left(c^{t} x, c^{t} x^{0}\right)$. The researchers define the dual problem as follows:

$$
\begin{aligned}
& \widetilde{\min _{y}} b^{t} y \\
& \text { s.t. } \quad A^{t} y \succeq c \\
& \quad y \geq 0
\end{aligned}
$$

After transforming the objective to a constraint with aspiration level $b^{t} y^{0}$, this problem becomes

$$
\begin{aligned}
& b^{t} y \preceq b^{t} y^{0} \\
& A^{t} y \succeq c \\
& y \geq 0 .
\end{aligned}
$$

The crisp linear problem analog to $\left(P 1 b^{\prime}\right)$ is then

$$
\begin{array}{ll} 
& \min _{y, \kappa}-\kappa \\
\text { s.t. } & \mu_{\preceq}\left(b^{t} y, b^{t} y^{0}\right) \geq \kappa \\
& \mu_{\succeq}\left(A^{t} y, c\right) \geq \kappa \\
& \kappa \in[0,1] \\
& y \geq 0
\end{array}
$$

with a solution $(y, \kappa)$.

Note that $\mu_{\succeq}\left(A^{t} y, c\right)$ is not a single number, but rather a vector containing, for each constraint $j$, the value $\mu_{\succeq(j)}\left(\left(A^{t}\right)_{j} y, c_{j}\right)$, where $\mu_{\succeq(j)}$ stands for the corresponding membership function. Let the tolerance interval of the membership function $\mu_{\succeq(j)}$ be $q_{j}$ (see (8)) and $q=\left(q_{1}, \ldots, q_{n}\right)$. Let, in addition $q_{0}$ be the tolerance interval of the membership function $\mu_{\preceq}\left(b^{t} y, b^{t} y^{0}\right)$. 
Weak duality For each feasible solution $(x, \alpha)$ of $\left(P 1 b^{\prime}\right)$ and each feasible solution $(y, \kappa)$ of $\left(D 1 b^{\prime}\right)$ the following inequality holds:

$$
(\alpha-1) p^{t} y-(\kappa-1) q^{t} x \leq\left(b^{t} y-c^{t} x\right)
$$

The theorem is equivalent to the crisp weak duality theorem in case $\alpha=\kappa=1$. Bector and Chandra [3] additionally show that, given two feasible solutions $(x, \alpha)$ and $(y, \kappa)$ of $\left(P 1 b^{\prime}\right)$ and $\left(D 1 b^{\prime}\right)$, respectively, the following implication holds:

If the subsequent conditions hold

$-(\alpha-1) p^{t} y+(\kappa-1) q^{t} x=\left(b^{t} y-c^{t} x\right)$

- $(\alpha-1) p_{0}+(\kappa-1) q_{0}=\left(c^{t} x-b^{t} y\right)+\left(b^{t} y^{0}-c^{t} x^{0}\right)$

$-c^{t} x^{0}-b^{t} y^{0} \leq 0$,

then $(x, \alpha)$ and $(y, \kappa)$ are optimal solutions.

In this subsection, we would also like to mention the paper by Gupta and Mehlawat [25]. It follows an approach that is very similar to that of Bector and Chandra [3] and that differs in the type of the membership functions (exponential membership functions) for the constraints and objectives of $(P 1 b)$ and $(D 1 b)$. The researchers provide the same duality results with a modification for the particular membership functions. In addition, Gupta and Mehlawat [25] show how their approach can be applied to problems of class 31 by using ranking functions for the comparison of fuzzy numbers and transforming the fuzzy problems of class 31 to the problems $\left(P 1 b^{\prime}\right)$ and $\left(D 1 b^{\prime}\right)$. Due to the similarities of the ideas in the papers by Bector and Chandra [3] and Gupta and Mehlawat [25], we omit a detailed presentation of the results of Gupta and Mehlawat [25].

\subsubsection{Approach of Hamacher et al. (1978)}

The paper by Hamacher et al. [26] considers a primal problem with fuzzy maximization and a mixture of fuzzy and crisp constraints. Since crisp contraints can be seen as a special case of fuzzy constraints, we consider the paper in this class. The primal problem is defined as follows:

$$
\begin{array}{ll}
\widetilde{\max _{x}} & c^{t} x \\
\text { s.t. } & A x \preceq b \\
& D x \leq f \\
& x \geq 0
\end{array}
$$

Similar to the other two approaches presented in this section, $\widetilde{\max }$ is defined by the membership function $\mu_{\succ}\left(c^{t} x, c^{t} x^{0}\right)$, where $c^{t} x^{0}$ is the aspiration level of the decision maker. $\preceq$ is defined by the membership function $\mu_{\preceq}(A x, b)$ representing soft constraints. Following the symmetrical approach of Bellman and Zadeh [4] and the assumption of linear membership functions, the researchers rewrite $(P 1 c)$ as the fol- 
lowing crisp linear optimization problem:

$$
\begin{array}{ll} 
& \max _{x, \alpha} \alpha \\
\text { s.t. } & \mu_{\succeq}\left(c^{t} x, c^{t} x^{0}\right) \geq \alpha \\
& \mu_{\preceq}(A x, b) \geq \alpha \\
& D x \leq f \\
& x \geq 0 \\
& \mu_{\succeq}\left(c^{t} x, c^{t} x^{0}\right) \in[0,1] \\
& \mu_{\preceq}(A x, b) \in[0,1]
\end{array}
$$

The dual problem is defined as the crisp dual of $\left(P 1 c^{\prime}\right)$, which is also a crisp linear problem and strongly depends on the membership functions chosen. The researchers provide an economic interpretation of the variables of the dual problem and admit that as opposed to the crisp case not all of them can be interpreted as shadow prices. Thus, they perform sensitivity analysis on the primal problem to "... derive the functional relationships between changes of components of the right-hand-side and changes of the optimal value of the primal objective functions ...” (p. 270). The researchers do not prove any duality results.

\subsubsection{Summary}

To sum up, the paper by Rödder and Zimmermann [63] aims to define fuzzy duality in a very intuitive way, using a clear economic interpretation. However, the approach suffers from some disadvantages, such as the particular form of the membership functions, which allow values higher than one and lower than zero. In addition, the researchers show only weak duality, which they prove in a mathematically very restricted setting.

The paper by Bector and Chandra [3] modifies the ideas in Rödder and Zimmermann [63] to address most of the above mentioned disadvantages. In addition, here the optimization problems are easier to solve than in [63]. However, there are also a few disadvantages. First, the approach is restricted to the use of linear monotone membership functions. Secondly, they prove only weak duality and provide a criterion to test solutions for optimality. However, Bector and Chandra [3] admit that strong duality would generally not hold.

Finally, the paper by Hamacher et al. [26] extends the literature on duality theory in fuzzy linear optimization by performing sensitivity analysis on the primal problem.

\subsection{Class 8: Fuzzy constraints}

The primal optimization problem in this class is

$$
\begin{array}{ll}
\max _{x} c^{t} x \\
\text { s.t. } & A x \preceq b \\
& x \geq 0 .
\end{array}
$$


Note that here only the fuzzy order operator must be defined. We found only one paper [77] that addresses duality of this problem type. The researcher defines $\preceq$ similarly to Bector and Chandra [3] through the use of a continuous and strictly monotone

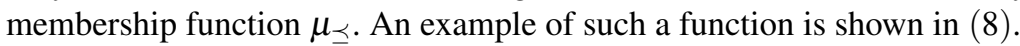

The difference to problems of class 24 (fuzzy maximization and fuzzy constraints) is that here the symmetric principle of Bellman and Zadeh [4] cannot be applied as max is a crisp operator and not a fuzzy one and thus the objective and the constraints cannot be treated symmetrically. Therefore, Verdegay [77] suggests a parametric approach where the degree to which the constraints of $(P 2)$ are satisfied is represented by an exogenously given parameter $\alpha$. $(P 2)$ can then be transformed into:

$$
\begin{array}{ll}
\max _{x} & c^{t} x \\
\text { s.t. } & \mu_{\preceq}(A x, b) \geq \alpha \\
& \alpha \in[0,1] \\
& x \geq 0 .
\end{array}
$$

This results in a fuzzy set as an optimal value because, for each degree of certainty $\alpha$, we obtain a different solution $x$.

The dual problem in reference [77] is defined as

$$
\begin{array}{ll}
\widetilde{\min _{y}} & \widetilde{b}^{t} y \\
\text { s.t. } & A^{t} y \geq c \\
& y \geq 0,
\end{array}
$$

which has the same fuzzy components as the problems of class 18. (D2) is transformed into a parametric problem with an exogeneously given parameter $\beta$ as follows:

$$
\begin{array}{ll}
\min _{y} & b^{t} y \\
\text { s.t. } & \mu_{j}\left(b_{j}\right) \geq 1-\beta, j \in 1, . ., m \\
& A^{t} y \geq c \\
& y \geq 0 \\
& \beta \in[0,1] .
\end{array}
$$

where, $\mu_{j}$ are membership functions for the coefficients of the objective. However, it is not clear from the paper why the equivalence between $(D 2)$ and $\left(D 2^{\prime}\right)$ holds. The interested reader should turn to [24, pp. 231ff] for more information. For continuous and strictly monotone membership functions, $\left(D 2^{\prime}\right)$ can be transformed to

$$
\begin{array}{ll}
\min _{y} & \sum_{j} \mu_{j}^{-1}(1-\beta) y_{j} \\
\text { s.t. } & A^{t} y \geq c \\
& y \geq 0 \\
& \beta \in[0,1] .
\end{array}
$$

Here, in contrast to $\left(P 2^{\prime}\right)$, the parameter $\beta$ does not affect the feasible region, but only the value of the objective function.

Strong duality Given $P 2^{\prime}$ (resp. $D 2^{\prime}$ ) with continuous and strictly monotone membership functions $\mu_{\preceq}$ (resp. $\mu_{j} \forall j$ ) and the corresponding dual problem problem $D 2^{\prime}$ (resp. $P 2^{\prime}$ ), the optimal objective values are the same fuzzy sets. The proof is based on crisp duality theory. 
An advantage of this approach is that it applies very elegantly the main reason for the popularity of duality theory, namely that by solving one of the primal or dual problems we can find the solution of the other. In this case, the primal problem is very difficult to solve as it contains the parameter $\alpha$ in each of its constraints, i.e. the parameter $\alpha$ determines the feasible region. The problem (D2) on the other hand can be transformed into a crisp linear problem, where the parameter $\beta$ is only part of the objective function. This makes (D2) much easier to solve. Note that the paper can just as well be classified in class 18 since all the results hold also when the primal problem is $(D 2)$. Then the dual problem would be $(P 2)$.

3.3 Class 18: Fuzzy maximization, fuzzy coefficients of objective function

The primal problem in this section is

$$
\begin{array}{ll}
\widetilde{\max _{x}} & \widetilde{c}^{t} x \\
\text { s.t. } & A x \leq b \\
& x \geq 0 .
\end{array}
$$

We found only one paper that deals with this type of primal problem [7]. The researchers work with general fuzzy numbers. Note that this problem is equivalent (in terms of parts that are fuzzified) to the problem (D2) presented in subsection 3.2. Cadenas Figueredo and Jiménez Barrionuevo [7] follow the approach in Subsection 3.2 and rewrite $(P 3)$ as a parametric problem with an exogeneously given parameter $\alpha$ :

$$
\begin{array}{ll}
\max _{x} & c^{t} x \\
\text { s.t. } & \mu_{j}\left(c_{j}\right) \geq \alpha, j \in 1, \ldots, n \\
& A x \leq b \\
& x \geq 0 \\
& \alpha \in[0,1]
\end{array}
$$

where the membership function $\mu_{j}\left(c_{j}\right)$ is non-negative on the interval $\left[c_{j}-d_{j}^{1}, \overline{c_{j}}+\right.$ $\left.d_{j}^{2}\right] \forall j$ and equals to one in the interval $\left[c_{j}, \overline{c_{j}}\right]$. Problem $\left(P 3^{\prime}\right)$ is then equivalent to the following interval parameter linear programming problem:

$$
\begin{array}{ll}
\max _{x} & {\left[\underline{c}-d^{1} \alpha, \bar{c}+d^{2} \alpha\right] x} \\
\text { s.t. } & A x \leq b \\
& x \geq 0 \\
& \alpha \in[0,1],
\end{array}
$$

where $\underline{c}=\left(\underline{c_{1}}, \ldots, \underline{c_{n}}\right), \bar{c}=\left(\overline{c_{1}}, \ldots, \overline{c_{n}}\right), d^{1}=\left(d_{1}^{1}, \ldots, d_{n}^{1}\right), d^{2}=\left(d_{1}^{2}, \ldots, d_{n}^{2}\right)$.

In the next step the researchers derive the crisp dual problem of $\left(P 3^{\prime \prime}\right)$ as follows:

$$
\begin{array}{ll}
\min _{y} & b^{t} y \\
\text { s.t. } & A^{t} y \geq\left[\underline{c}-d^{1} \alpha, \bar{c}+d^{2} \alpha\right] \\
& y \geq 0 \\
& \alpha \in[0,1]
\end{array}
$$


Let

$$
\begin{aligned}
& K_{\text {min }}=\left\{y \geq 0 \mid A^{t} y \geq \underline{c}-d^{1} \alpha\right\} \\
& K=\left\{y \geq 0 \mid A^{t} y \geq c^{*}, c^{*} \in\left[\underline{c}-d^{1} \alpha, \bar{c}+d^{2} \alpha\right]\right\} \\
& K_{\text {max }}=\left\{y \geq 0 \mid A^{t} y \geq \bar{c}+d^{2} \alpha\right\}
\end{aligned}
$$

Then $K_{\max } \subset K \subset K_{\min }$. The researchers use these relationships to show that the optimal value of problem (12) is bounded from above and below by the optimal values of the following two auxiliary crisp linear parametric problems:

$$
\begin{array}{ll}
\min _{y} & b^{t} y \\
\text { s.t. } & A^{t} y \geq \underline{c}-d^{1} \alpha \\
& y \geq 0 \\
& \alpha \in[0,1] \\
& \min _{y} b^{t} y \\
\text { s.t. } & A^{t} y \geq \bar{c}+d^{2} \alpha \\
& y \geq 0 \\
& \alpha \in[0,1]
\end{array}
$$

Based on the duality between $\left(P 3^{\prime \prime}\right)$ and (12), the researchers show that, if $y_{\min }(\alpha)$ is the optimal solution of $(A 1)$ and $y_{\max }(\alpha)$ the optimal solution of $(A 2)$, then the optimal value of the objective function of $\left(P 3^{\prime \prime}\right)$ for a given $\alpha \in[0,1]$ is included in the interval:

$$
\left[b^{t} y_{\min }(\alpha), b^{t} y_{\max }(\alpha)\right]
$$

The dual problem in this subsection is defined as:

$$
\begin{array}{ll}
\min _{y} & b^{t} y \\
\text { s.t. } & A^{t} y \succeq c \\
& y \geq 0 .
\end{array}
$$

Note that by choosing suitable membership functions, both $(A 1)$ and $(A 2)$ can be rewritten in the form of $(D 3)$. Thus, it can be shown that the optimal value of the objective function of $(P 3)$ is bounded by the optimal values of the objective functions of two fuzzy linear optimization problems of type (D3). Similarly, the researchers prove that the optimal value of the objective function of an optimization problem of type $(D 3)$ is bounded by the optimal value of the objective functions of two fuzzy optimization problems of type $(P 3)$.

Strong duality Given a problem $(P 3)$, there always exist two problems of type $(D 3)$ so that the optimal value of the objective function of $(P 3)$ is both lower and upper bounded by the optimal values of the objective functions of the problems of type (D3). Analogously, given a problem of type (D3), there always exist two problems of type $(P 3)$ so that the the optimal value of the objective function of $(D 3)$ is both lower and upper bounded by the optimal values of the objective functions of the problems of type $(P 3)$.

To sum up, the paper by Cadenas Figueredo and Jiménez Barrionuevo [7] uses linear interval programming to define the concept of duality. The approach is very intuitive and quite innovative. Moreover, the results in this paper extend and systematize the work of Verdegay [77]. However, the researchers do not present any other duality theorems except strong duality. 
3.4 Class 31: Fuzzy maximization, fuzzy constraints, fuzzy coefficients of constraints, fuzzy limit values of constraints and fuzzy coefficients of objective function

The primal fuzzy linear optimization problem in this class is defined as follows:

$$
\begin{array}{ll}
\widetilde{\max _{x}} & \widetilde{c}^{t} x \\
\text { s.t. } & \widetilde{A} x \preceq \widetilde{b} \\
& x \geq 0
\end{array}
$$

In order to properly define problem (P4), we need to define which fuzzy numbers are used and how the operators " $\preceq$ " and " $\widetilde{\max }$ " are specified. The literature [60, $31,61,87,46,62]$ is not homogeneous in this regard, which results in syntactically similar, but semantically different duality theorems. Thus, for each of the papers we explicitly present the respective results of the researchers and finally provide a brief overview of the approaches at the end of this subsection.

\subsubsection{Approach of Inuiguchi et al. (2003)}

The works by Ramík [60] and Inuiguchi et al. [31] follow a very similar approach, therefore we discuss only the second one here. Inuiguchi et al. [31] use compact, strictly convex, normal fuzzy subsets of the real numbers. They define $\preceq$ as a fuzzy relation with a membership function $\mu_{\preceq}: F(\mathbb{R}) \times F(\mathbb{R}) \rightarrow[0,1]$, which is based on t-norms, t-conorms and valued relations. Inuiguchi et al. [31] define $\widetilde{\max }$ using an exogenously given aspiration level $\widetilde{d} \in F(\mathbb{R})$ and the fuzzy relation $\preceq$, in which their approach differs from the approaches in the other papers presented in this subsection. The researchers rewrite $(P 4)$ as follows:

$$
\begin{aligned}
& \tilde{c}^{t} x \succeq \tilde{d} \\
& \widetilde{A} x \preceq \widetilde{b} \\
& x \geq 0
\end{aligned}
$$

Inuiguchi et al. [31] define the set of $\alpha$-feasible solutions ${ }^{6}, 0 \leq \alpha \leq 1$, as those $x \geq 0$ which satisfy the constraints of (P4) (i.e. $\widetilde{A} x \preceq \widetilde{b}, x \geq 0$ in $(P 4 a)$ ) at least with degree $\alpha$. An $\alpha$-satisficing solution is an $\alpha$-feasible solution $x$ that additionally satisfies the objective of $(P 4)$ (i.e. the constraint $\widetilde{c}^{t} x \succeq \widetilde{d}$ in $(P 4 a)$ ) at least with degree $\alpha$. This concept is based on the symmetric approach of Bellman and Zadeh [4].

The dual problem in [31] is defined as follows:

$$
\begin{aligned}
& \widetilde{b}^{t} y \preceq \widetilde{h} \\
& \widetilde{A}^{t} y \succeq \widetilde{c} \\
& y \geq 0
\end{aligned}
$$

\footnotetext{
${ }^{6}$ Note that we define the concepts of $\alpha$-feasible and $\alpha$-satisficing solution here for the particular case to which the researchers apply duality theory. Their definition is much broader.
} 
Weak duality Under some assumptions (p. 171), for each $\alpha$-feasible solution $x$ of $(P 4 a)$ and each $\alpha$-feasible solution $y$ of $(D 4 a)$ the following inequality holds:

$$
\sum_{j}{\widetilde{c_{j}}}^{R}(1-\alpha) x_{j} \leq \sum_{i}{\widetilde{b_{i}}}^{R}(1-\alpha) y_{i} \text { for } \alpha \in[0.5,1)
$$

They call property (13) "a weak form of the duality theory". Here, $\widetilde{c}_{j}^{R}(1-\alpha)$ and $\widetilde{b}_{i}^{R}(1-\alpha)$ are the upper bounds of the " $(1-\alpha)-$ cuts" of $\widetilde{c_{j}}$ and $\widetilde{b_{i}}$, respectively, i.e. ${\widetilde{c_{j}}}^{R}(1-\alpha)=\sup \widetilde{c_{j}}[1-\alpha]$ and ${\widetilde{b_{i}}}^{R}(1-\alpha)=\sup \widetilde{b_{i}}[1-\alpha]$.

Strong duality If there exists an $\alpha$-satisficing solution for $(P 4 a)$ and a $(1-\alpha)$ satisficing solution for $(D 4 a)$, then and under some additional assumptions (p. 172) there exist an $\alpha$-satisficing solution $x^{*}$ for $(P 4 a)$ and a $(1-\alpha)$-satisficing solution $y^{*}$ for $(D 4 a)$ such that

$$
\sum_{j}{\widetilde{c_{j}}}^{R}(\alpha) x_{j}=\sum_{i}{\widetilde{b_{i}}}^{R}(\alpha) y_{i}
$$

\subsubsection{Approach of Ramík (2005)}

The approach of Ramík [61] modifies the approach of Inuiguchi et al. [31] ${ }^{7}$ Moreover, the ideas of Ramík [62] can be seen as an extension of the ideas of Ramík [61]. While Ramík [61] proves duality results only for the case, where the comparison operators in the primal problem are based on t-norms and those in the dual are based on t-conorms, Ramík [62] proves also weak and strong duality for the opposite case. We thus believe that presenting the ideas of Ramík [61] in detail is enough to understand both papers.

Ramík [61] similarly to Inuiguchi et al. [31] defines $\preceq$ as a fuzzy relation, which is based on t-norms, t-conorms and valued relations. In contrast to Inuiguchi et al. [31], in order to determine $\widetilde{\max }$, [61] does not transform the objective into a constraint. Instead he uses the following relation on the set of fuzzy numbers, which is a binary relation for a given $\alpha \in(0,1]$ and fuzzy numbers $\widetilde{a}$ and $\tilde{b}$ :

$$
\widetilde{a} \prec_{\alpha} \widetilde{b} \Leftrightarrow\left(\mu_{\preceq}(\widetilde{a}, \widetilde{b}) \geq \alpha \wedge \mu_{\preceq}(\widetilde{b}, \widetilde{a})<\alpha\right)
$$

An $\alpha$-efficient solution for $(P 4)$ (i.e. definition of $\widetilde{\max }$ ) is then defined as an $\alpha$ feasible solution $x$ such that there does not exist an $\alpha$-feasible solution $x^{\prime}$ with $\tilde{c}^{t} x \prec_{\alpha}$ $\widetilde{c}^{t} x^{\prime}$.

Ramík [61] defines the dual problem as follows:

$$
\begin{array}{ll}
\widetilde{\min _{y}} & \widetilde{b}^{t} y \\
\text { s.t. } & \widetilde{A}^{t} y \succeq \widetilde{c} \\
& y \geq 0
\end{array}
$$

where the comparison operator $\succeq$ is the dual operator of $\preceq$ Ramík [61, p. 26f.], as used in the primal problem (P4).

\footnotetext{
7 The researcher, too, works with the same type of fuzzy numbers as Inuiguchi et al. [31] with the difference that the membership functions of Ramík [61] are semistrictly quasi-concave.
} 
Weak duality Ramík [61] defines two weak duality theorems. The first one states that under some assumptions (p. 33f) for an $\alpha$-feasible solution $x$ of (P4) and a $(1-\alpha)$-feasible solution $y$ of $(D 4 b)$ the following inequality holds:

$$
\sum_{j}{\widetilde{c_{j}}}^{R}(\alpha) x_{j} \leq \sum_{i} \widetilde{b}_{i}^{R}(\alpha) y_{i}
$$

where $\widetilde{c}_{j}^{R}(\alpha)$ and $\widetilde{b}_{i}^{R}(\alpha)$ are defined as in subsection 3.4.1.

Regarding the second weak duality theorem, the researcher proves that if

$$
\sum_{j}{\widetilde{c_{j}}}^{R}(\alpha) x_{j}=\sum_{i}{\widetilde{b_{i}}}^{R}(\alpha) y_{i}
$$

then $x$ is $\alpha$-efficient for $(P 4)$ and $y$ is $(1-\alpha)$-efficient for (D4b). This theorem can be used as a test criterion for optimality.

Strong duality Under some assumptions (p. 35) (P4) has an $\alpha$-efficient solution $x^{*}$ and $(D 4 b)$ has a $(1-\alpha)$-efficient solution $y^{*}$ and the following equation holds:

$$
\sum_{j}{\widetilde{c_{j}}}^{R}(\alpha) x_{j}=\sum_{i}{\widetilde{b_{i}}}^{R}(\alpha) y_{i}
$$

\subsubsection{Approach of $W u(2003)$}

The researcher [87] works with normal, convex fuzzy subsets of the real numbers with upper-semicontinuous membership functions and compact $\alpha$-cut for $\alpha=0$. He defines $\succeq$ for two fuzzy numbers $\widetilde{a}$ and $\widetilde{b}$ as follows:

$$
\widetilde{a} \succeq \widetilde{b}: \Leftrightarrow\left(\widetilde{a}(\alpha)^{L} \geq \widetilde{b}(\alpha)^{L} \wedge \widetilde{a}(\alpha)^{R} \geq \widetilde{b}(\alpha)^{R} \forall \alpha \in[0,1]\right)
$$

Here $\widetilde{a}(\alpha)^{L}$ is the lower bound of the " $\alpha-$ cut" of $\widetilde{a}$. Note that $\succeq$ is a binary relation on the set of fuzzy numbers, as it is not parametrized by $\alpha$.

In contrast to the approaches of Inuiguchi et al. [31] and Ramík [61], this paper defines the primal problem as a minimization problem and the dual problem as a maximization problem:

$$
\begin{array}{ll}
\widetilde{\min _{x}} & \widetilde{c}^{t} x \\
\text { s.t. } & \widetilde{A} x \succeq \widetilde{b} \\
& x \geq 0 \\
\widetilde{\max _{y}} & \widetilde{b}^{t} y \\
\text { s.t. } \quad & \widetilde{A}^{t} y \preceq \widetilde{c} \\
& y \geq 0
\end{array}
$$

The researchers define the optimal solution of $(P 4 c)$ as the feasible solution $x^{*}$ that minimizes $\widetilde{c}^{t} x$ over all feasible solutions according to the definition of $\succeq$. The optimal solution of $(D 4 c)$ is defined analogously. 
Weak duality For each feasible solution $x$ of $(P 4 c)$ and each feasible solution $y$ of $(D 4 c)$, the following inequality holds:

$$
\widetilde{c}^{t} x \succeq \widetilde{b}^{t} y
$$

In addition, the researcher shows that if (23) holds with equality for two feasible solutions of the primal and the dual problem, then these solutions are optimal.

Strong duality Even though the comparison operator defined in $\mathrm{Wu}$ [87] is a binary relation, it must hold $\forall \alpha \in[0,1]$. This makes it difficult to prove strong duality analogously to the crisp case. The researchers solve this problem by transforming $(P 4 c)$ and $(D 4 c)$ into the following four crisp linear parametric problems:

$$
\begin{aligned}
& \min _{x}\left(\widetilde{c}_{\alpha}^{L}\right)^{t} x \\
& \text { s.t. } \widetilde{A}_{\alpha}^{L} x \geq \widetilde{b}_{\alpha}^{L} \quad\left(P 4 c_{\alpha}^{L}\right) \\
& x \geq 0 \\
& \min _{x}\left(\widetilde{c}_{\alpha}^{R}\right)^{t} x \\
& \text { s.t. } \widetilde{A}_{\alpha}^{R} x \geq \widetilde{b}_{\alpha}^{R} \quad\left(P 4 c_{\alpha}^{R}\right) \\
& x \geq 0 \\
& \max _{y}\left(\widetilde{b}_{\alpha}^{L}\right)^{t} y \\
& \text { s.t. }\left(\widetilde{A}_{\alpha}^{L}\right)^{t} y \leq \widetilde{c}_{\alpha}^{L} \quad\left(D 4 c_{\alpha}^{L}\right) \\
& y \geq 0 \\
& \max _{y}\left(\widetilde{b}_{\alpha}^{R}\right)^{t} y \\
& \text { s.t. }\left(\widetilde{A}_{\alpha}^{R}\right)^{t} y \leq \widetilde{c}_{\alpha}^{R} \quad\left(D 4 c_{\alpha}^{R}\right) \\
& y \geq 0
\end{aligned}
$$

Note that, for fixed $\alpha,\left(P 4 c_{\alpha}^{L}\right)$ and $\left(D 4 c_{\alpha}^{L}\right)$ as well as $\left(P 4 c_{\alpha}^{R}\right)$ and $\left(D 4 c_{\alpha}^{R}\right)$ are dual pairs, for which crisp duality theory holds. The researcher defines further the following sets:

$$
\begin{aligned}
& A R G M I N_{P_{\alpha}^{L}}=\text { set of all finite optimal solutions of }\left(P 4 c_{\alpha}^{L}\right) \\
& \operatorname{ARGMIN}_{P_{\alpha}^{R}}=\text { set of all finite optimal solutions of }\left(P 4 c_{\alpha}^{R}\right) \\
& \operatorname{ARGMIN}_{P_{L}}=\cap_{0 \leq \alpha \leq 1}\left(A R G M I N_{P_{\alpha}^{L}}\right) \\
& \operatorname{ARGMIN}_{P_{R}}=\cap_{0 \leq \alpha \leq 1}\left(\operatorname{ARGMIN}_{P_{\alpha}^{R}}\right) \\
& \operatorname{ARGMIN}_{P}=A R G M I N_{P_{L}} \cap A R G M I N_{P_{R}}
\end{aligned}
$$

Intuitively, $A R G M I N_{P_{L}}$ contains the lower bound $\alpha$-cut solutions of the primal problem, that hold for each $0 \leq \alpha \leq 1$. Analogously, $A R G M I N_{P_{R}}$ contains the upper bound $\alpha$-cut solutions of the primal problem, that hold for each $0 \leq \alpha \leq 1$. $A R G M I N_{P}$ contains the optimal solutions that cover both the lower and the upper bound of the primal problem. Since a fuzzy number can be represented through its $\alpha$-cuts, we would expect that $A R G M I N_{P}$ covers the optimal solutions of $(P 4 c)$. Analog notations are used for the dual problem, where $A R G M I N_{D}$ would cover the optimal solutions for $(D 4 c)$.

The strong duality theorem then states that if $A R G M I N_{P}$ and $A R G M I N_{D}$ are 
nonempty, then $(P 4 c)$ and $(D 4 c)$ have no duality gap.

Complementary slackness Suppose that $x^{*}$ and $y^{*}$ are feasible solutions of (P4c) and (D4c), respectively. If one of the following conditions is satisfied

$$
\begin{gathered}
\left(\widetilde{A} x^{*}=\widetilde{b}\right) \wedge\left(\widetilde{c}^{t} x^{*}=\left(\widetilde{A} x^{*}\right)^{t} y^{*}\right) \\
\left(\widetilde{A}^{t} y^{*}=c\right) \wedge\left(\widetilde{b}^{t} y^{*}=\left(\widetilde{A^{t}} y^{*}\right)^{t} x^{*}\right) \\
\left(\widetilde{c}^{t} x^{*}=\left(y^{*}\right)^{t}\left(\widetilde{A} x^{*}\right)\right) \wedge\left(\widetilde{b}^{t} y^{*}=\left(\widetilde{A}^{t} y^{*}\right)^{t} x^{*}\right) \\
\left(\widetilde{c}^{t} x^{*}=\left(\widetilde{A}^{t} y^{*}\right)^{t} x^{*}\right) \wedge\left(\widetilde{b}^{t} y^{*}=\left(y^{*}\right)^{t}\left(\widetilde{A} x^{*}\right)\right),
\end{gathered}
$$

then $x^{*}$ and $y^{*}$ are optimal solutions of (P4c) and (D4c), respectively.

\subsubsection{Approach of Mahdavi-Amiri and Nasseri (2006)}

Mahdavi-Amiri and Nasseri [46] work with trapezoidal fuzzy numbers and define $\succeq$ using linear ranking functions. The researchers define the optimal solution of $(P 4)$ as the feasible solution with the highest objective value according to the definition of $\succeq$. The dual problem is defined as

$$
\begin{array}{ll}
\min _{y} \widetilde{b}^{t} y \\
\text { s.t. } \quad \widetilde{A}^{t} y \succeq \widetilde{c} \\
\quad y \geq 0 .
\end{array}
$$

Weak duality For each feasible solution $x$ of $(P 4)$ and each feasible solution $y$ of $(D 4 d)$ the following holds:

$$
\widetilde{c}^{t} x \preceq \widetilde{b}^{t} y
$$

In addition, the researchers show that if $\widetilde{c}^{t} x \approx \widetilde{b}^{t} y$, then the solution $x$ is optimal for $(P 4)$ and the solution $y$ is optimal for $(D 4 d)$. This can be used as a test criterion for optimality.

Strong duality If one of $(P 4)$ or $(D 4 d)$ has an optimal solution $\left(x^{*}\right.$ resp. $\left.y^{*}\right)$, then both problems have optimal solutions and $\widetilde{c}^{t} x^{*} \approx \widetilde{b}^{t} y^{*}$.

Complementary slackness Given two feasible solutions $x^{*}$ of $(P 4)$ and $y^{*}$ of $(D 4 d)$, they are optimal if and only if

$$
\left(\widetilde{A}^{t} y^{*}-\widetilde{c}\right)^{t} x^{*}+\left(y^{*}\right)^{t}\left(\widetilde{b}-\widetilde{A} x^{*}\right) \approx 0 .
$$

Fundamental theorem of duality For $(P 4)$ and $(D 4 d)$ exactly one of the following holds:

1. Both have optimal solutions $x^{*}$ and $y^{*}$ and $\widetilde{c}^{t} x^{*} \approx \widetilde{b}^{t} y^{*}$.

2. One problem is unbounded and the other is infeasible.

3. Both problems are infeasible. 


\subsubsection{Summary}

We now discuss the findings of the papers presented in this section. The paper by Inuiguchi et al. [31] defines fuzzy optimization similar to the symmetric approach by Bellman and Zadeh [4], requiring the definition of fuzzy goals. The researchers prove both weak and strong duality. The main contribution of the paper is that it defines fuzzy order as well as maximization in terms of membership functions and thus retains the initial fuzzy uncertainty in the operators.

Ramík [61] extends the paper by Inuiguchi et al. [31] in that he defines fuzzy maximization in a more natural way (using the highest value and not fuzzy goals). His duality results are also more general.

The paper by $\mathrm{Wu}$ [87] provides a duality theory that defines the comparison of fuzzy numbers using a binary relation. The disadvantage of the approach is that thereby the uncertainty stemming from the fuzzy order is lost as the comparison between two fuzzy number is only a "true" or "false" decision and not a fuzzy set.

Finally, the paper by Mahdavi-Amiri and Nasseri [46] presents for each crisp dualiy theorem a fuzzy duality theorem. However, they can prove those theorems only because of the use of ranking functions, which allows them to do all the proofs analogously to the crisp case. This results again to loss of uncertainty in the comparison operators. Moreover, the used trapezoidal fuzzy numbers need to be additionally restricted to a zero left (right) spread as discussed in the introduction of Section 3.

\subsection{Fuzzy decision variables}

In this subsection, we discuss papers that cover FLP with fuzzy decision variables. As explained in the introductory part of this section, these approaches have limited practical usefulness. However, since the approaches represent a significant part of fuzzy duality theory literature, we believe that they should be discussed here for the reason of completeness of theoretical approaches.

3.5.1 Class 30: Fuzzy maximization, fuzzy constraints, fuzzy coefficients of objective function, fuzzy decision variables

The primal problem in this class is

$$
\begin{array}{ll}
\widetilde{\max _{\tilde{x}}} & \widetilde{c} \widetilde{x} \\
\text { s.t. } & A \widetilde{x} \preceq \widetilde{b} \\
& \widetilde{x} \succeq \widetilde{0} .
\end{array}
$$

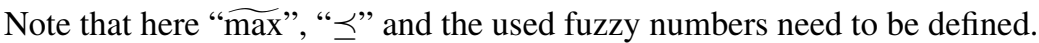

The paper [50] considers $(P 5 a)$ as its primal problem and the researchers derive their results using symmetric trapezoidal numbers. For the comparison of fuzzy numbers they apply a linear ranking function. The optimal solution of $(P 5 a)$ is defined as the feasible solution with the highest objective value according to the defined fuzzy 
comparison operator. The dual problem is defined as

$$
\begin{array}{ll}
\widetilde{\min _{\tilde{y}}} \widetilde{b}^{t} \widetilde{y} \\
\text { s.t. } & A^{t} \widetilde{y} \succeq \widetilde{c} \\
& \widetilde{y} \succeq 0 .
\end{array}
$$

Note that the solutions of $(P 5 a)$ and $(D 5 a)$ are fuzzy numbers.

Weak duality For two feasible solutions $\widetilde{x}$ and $\tilde{y}$ of $(P 5 a)$ and $(D 5 a)$, respectively, the following inequality holds:

$$
\widetilde{b}^{t} \widetilde{y} \succeq \widetilde{c}^{t} \widetilde{x}
$$

Moreover, the researchers show that if $\widetilde{b}^{t} \widetilde{y} \approx \widetilde{c} \tau \tilde{x}$, then the feasible solutions are also optimal.

\subsubsection{Fully fuzzified linear problem (special case of class 31)}

The primal problem in this class looks as follows:

$$
\begin{array}{ll}
\widetilde{\max _{x}} & \widetilde{c} \widetilde{x} \\
\text { s.t. } & \widetilde{A} \widetilde{x} \preceq \widetilde{b} \\
& \widetilde{x} \succeq \widetilde{0}
\end{array}
$$

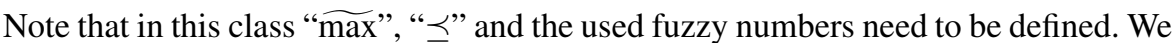
found only one paper by Hashemi et al. [28] that deals with $(P 5 b)$. The researchers work with symmetric LR fuzzy numbers ${ }^{8}$.

Hashemi et al. [28] propose a method for ordering fuzzy numbers which is similar to ranking functions. However, it differs in that the function (see p. 10) is defined as

$$
\begin{aligned}
& R: \mathbb{F}(\mathbb{R}) \rightarrow \mathbb{R}^{2} \\
& \widetilde{a} \mapsto[M(\widetilde{a}),-S D(\widetilde{a})] \\
& \widetilde{a} \succeq \widetilde{b} \Leftrightarrow R(\widetilde{a})-R(\widetilde{b}) \geq_{L G} 0 .
\end{aligned}
$$

Here, $M$ and $S D$ are the possibilistic mean and standard deviation of the fuzzy number and $\geq_{L G}$ denotes a "lexicographically non-negative vector". Note that, just as the other ranking functions, this function is a binary relation. The optimal solution of $(P 5 b)$ is the feasible solution with the highest objective value according to the definition of $\succeq$.

The researchers propose a two phase approach for solving $(P 5 b)$, where in the first phase they "... maximize the mean value of the fuzzy objective function subject to the constraints ..." (p. 257). The second phase is to "... minimize the standard deviation of the original fuzzy objective function subject to the set obtained at the first phase."

\footnotetext{
8 The researchers define fuzzy arithmetics based on interval arithmetic and $\alpha$-cuts, which is equivalent to the extension principle approach.
} 
(p. 257) In both phases crisp linear problems are solved.

The dual problem in this section is defined as

$$
\begin{array}{ll}
\widetilde{\min _{y}} & \widetilde{b}^{t} \widetilde{y} \\
\text { s.t. } & \widetilde{A^{t}} \widetilde{y} \succeq \widetilde{c} \\
& \widetilde{y} \succeq 0 .
\end{array}
$$

$(D 5 b)$ is solved analogously to $(P 5 b)$, by applying the dual operations to those in the primal problem. In the first phase, the mean of the objective function is minimized, while in the second phase "... among feasible solutions obtained at the end of the first phase the one with the maximum standard deviation is selected." (p. 259)

Weak duality For each feasible solution $\tilde{x}$ of $(P 5 b)$ and $\tilde{y}$ of $(D 5 b)$ it holds that $\widetilde{b}^{t} \widetilde{y} \succeq \widetilde{c}^{t} \widetilde{x}$. If in addition $\widetilde{b}^{t} \widetilde{y} \approx \widetilde{c}^{t} \widetilde{x}$, then the solutions are optimal.

\subsubsection{Summary}

In this subsection, we discuss the advantages and disadvantages of the two presented papers. Nasseri and Mahdavi-Amiri [50] prove only the weak duality theorem and their results are based on two very restrictive assumptions: (1) Fuzzy numbers are compared using linear ranking functions. (2) Fuzzy numbers are symmetric trapezoidal numbers. As discussed above, especially the second assumption is not appropriate in the context of FLP because $\widetilde{b}$ in $(P 5 a)$ and $\widetilde{c}$ in $(D 5 a)$ should be defined with a zero left and right spread respectively. The reason for this is that $\preceq$ and $\succeq$ should be satisfied in FLP below and above a given value with certainty, which is not the case for general symmetric trapezoidal numbers. However, their approach can be extended straightforward to asymmetric trapezoidal numbers with zero left and right spread. Thus, we recommend that the approach is only applied under this additional requirement.

Hashemi et al. [28] are quite innovative with their idea to use the possibilistic mean and standard deviation of fuzzy numbers. In addition, they provide a method to efficiently solve fully fuzzified optimization problems, which is a major contribution to fuzzy linear optimization. However, similar to Nasseri and Mahdavi-Amiri [50] their contribution to fuzzy duality theory is only a proof of the weak duality theorem.

\subsection{Other models}

In this section we consider more general models which do not fit in any of the above sections but if specified can cover some of the above classes. We identified two types of generalization: the first type refers to fuzzy linear multiple-criteria optimization problems $[44,93]$, the second type to fuzzy non-linear optimization problems $[39,67,86]$. While we present literature contributions of the former type in this subsection, we do not include literature contributions of the latter for the following reasons: a) An extension of our literature search with fuzzy non-linear optimization problems would extend this lengthy literature review substantially. We regard such work an independent review paper. b) Having reviewed the above fuzzy non-linear 
optimization papers we found that they draw on the Langragian approach and duality theory of non-linear optimization. Consistent with our overview of linear duality theory in the current paper, an overview of non-linear duality theory would then deem appropriate. However, such an overview would have extended this paper also. The interested reader might want to read our appendix where we present the ideas of (duality in) fuzzy non-linear optimization problems [39, 67, 86].

\subsubsection{Approach of Liu et al. (1995)}

Liu et al. [44] consider duality of fuzzy $M C^{2}$ (multiple criteria, multiple constraint levels) linear programming. The primal problem in this paper is defined as

$$
\begin{array}{ll}
\widetilde{\max _{x}} & \lambda^{t} C x \\
\text { s.t. } & A x \leq D \gamma \\
& x \geq 0,
\end{array}
$$

where $C$ and $D$ are matrices, $\lambda>0$ determines the weights of the different criteria and $\gamma>0$ determines the weights of constraints. Note that uncertainty in the above problem is included only in $\widetilde{\max }$. In case of one constraint and one objective, this problem belongs to class $16 . \widetilde{\max }$ is defined as a satisficing level between the lower and upper aspiration bound of the objective function.

To solve $(P 6 a)$ the researchers define the following two crisp optimization problems:

$$
\begin{array}{ll}
\max _{x} & \lambda^{t} C x \\
\text { s.t. } & A x \leq D \gamma \\
& x \geq 0 \\
\min _{x} & \lambda^{t} C x \\
\text { s.t. } & A x \leq D \gamma \\
& x \geq 0
\end{array}
$$

The researchers define the sets of all potential bases ${ }^{9}$ of $(A 3)$ and $(A 4)$, respectively, as $U=\left\{U_{1}, \ldots, U_{r}\right\}$ and $L=\left\{L_{1}, \ldots, L_{k}\right\}$. For every possible combination of elements of $U$ and $L$ and for each feasible solution $x$ of $(P 6 a)$ they define a linear membership function, which equals to one when the objective function of $(P 6 a)$ at $x$ is greater or equal to that of $(A 3)$ and equals to zero when the objective function of $(P 6 a)$ at $x$ is less or equal to that of $(A 4)$.

The dual problem is defined as follows:

$$
\begin{array}{ll}
\widetilde{\min _{y}} & \gamma D^{t} y \\
\text { s.t. } & A^{t} y \geq C^{t} \lambda \\
& u \geq 0
\end{array}
$$

Similarly to the primal problem, to solve the dual problem the researchers define two auxiliary crisp optimization problems with the same constraint set and objective function. The first auxiliary problem is a maximization problem and the second

\footnotetext{
9 The interested reader can refer to reference [44, p. 392, Definition 2.2] for more information on the potential basis of an $M C^{2}$ problem.
} 
is a minimization problem. A solution of $(D 6 a)$ is defined analogously to the primal problem by using a membership function over the solutions of the two auxiliary problems.

The researchers interpret fuzzy weak duality as the decision maker's preferences regarding $(P 6 a)$ and $(D 6 a)$.

Weak duality For a solution $x$ of $(P 6 a)$ with a membership function $\mu_{P}(x)$ and a solution $y$ of $(D 6 a)$ with a membership function $\mu_{D}(x)$, the relation between the two problems is defined as $\mu_{R}(x, y)=O\left(\mu_{P}(x), \mu_{D}(y)\right)$, where $O \in\{$ min, max, mean $\}$.

The reason to use a membership function as a basis for weak duality is that “... the decision makers want to identify a set of satisficing (fuzzy) solutions for the given problem, as well as its dual problem." (p. 410).

To sum up, the proposed approach provides a very elegant way for solving fuzzy $M C^{2}$ problems. However, Liu et al. [44] do not prove any other duality theorems except of weak duality.

3.6.2 Approach of Zhong and Shi (2002)

The paper of Zhong and Shi [93] also considers $M C^{2}$ problems, but the researchers propose a fuzzy duality theory different to that of Liu et al. [44]. Their idea follows that of Rödder and Zimmermann [63] as presented in subsection 3.1.1. They define the primal problem as follows:

$$
\begin{array}{ll}
\widetilde{\max _{x}} & \lambda^{t} C x \\
\text { s.t. } & A x \preceq D \gamma \\
& x \geq 0
\end{array}
$$

In case of one constraint and one objective this problem belongs to class 24 . $\widetilde{\max }$ is defined as a constraint using an aspiration level $z_{1}$. The primal problem can thus be rewritten as

$$
\begin{aligned}
& \lambda^{t} C x \succeq z_{1} \\
\text { s.t. } & A x \preceq D \gamma \\
& x \geq 0 .
\end{aligned}
$$

Note that, similar to [63], in an economic context the membership function of the constraints depends on the unit price $y$ of production resources on the market.

$\left(P 6 b^{\prime}\right)$ is solved using the symmetrical approach by Bellman and Zadeh [4] i.e. given the membership function $\mu_{\preceq}(A x, D \gamma, y)$ of the objective and $\mu_{\succeq}\left(\lambda^{t} C x, z_{1}\right)$ of the constraints, the solution is

$$
\max _{x \geq 0}\left(\min \left\{\mu_{\succeq}\left(\lambda^{t} C x, z_{1}\right), \mu_{\preceq}(A x, D \gamma, y)\right\}\right) .
$$

The dual problem is defined as

$$
\begin{array}{ll}
\widetilde{\min _{y}} & \gamma^{t} D^{t} y \\
\text { s.t. } & A^{t} y \succeq C^{t} \lambda \\
& y \geq 0
\end{array}
$$


and solved analogously.

To construct the membership functions of the constraints and objectives in the fuzzy primal and dual problems the researchers first solve their corresponding crisp problems, i.e

$$
\begin{array}{ll}
\max _{x} & \lambda^{t} C x \\
\text { s.t. } & A x \leq D \gamma \\
& x \geq 0 \\
& \\
\min _{y} & \gamma^{t} D^{t} y \\
\text { s.t. } & A^{t} y \geq C^{t} \lambda \\
& y \geq 0 .
\end{array}
$$

Then they determine, for each combination of potential solutions of (A5) and (A6), $(i, j)$, the intersection $R(i, j)$ of the primal and dual weight sets [93, p. 337, Def. 2.1] that satisfies given conditions ${ }^{10}$.

The membership functions of the primal and dual problem are then defined for each $R(i, j)$. We thus rewrite $(29)$ as:

$$
\max _{x \geq 0}\left(\min \left\{\mu_{\succeq}^{R(i, j)}\left(\lambda^{t} C x, z_{1}\right), \mu_{\preceq}^{R(i, j)}(A x, D \gamma, y)\right\}\right) .
$$

As an illustration, $\mu_{\succ}^{R(i, j)}\left(\lambda^{t} C x, z_{1}\right)$ equals to one at the potential solution of (A5) with $(\gamma, \lambda) \in R(i, j)$ and equals to zero at the optimal solution of the problem

$$
\begin{array}{ll}
\min _{x} & \lambda^{t} C x \\
\text { s.t. } & A x \leq D \gamma \\
& x \geq 0 \\
& (\gamma, \lambda) \in R(i, j),
\end{array}
$$

i.e. the aspiration level is the optimal objective value of the corresponding crisp $M C^{2}$ problem. Note that in this way, for each $R(i, j)$, there is one pair of primal and dual problems, which we call $(P 6 b)_{R(i, j)}$ and $(D 6 b)_{R(i, j)}$.

Weak duality Let $(\lambda, \gamma) \in R(i, j)$, and let $\left(\mu^{P(R(i, j))}\left(x^{*}\right), x^{*}\right)$ and $\left(\mu^{D(R(i, j))}\left(y^{*}\right), y^{*}\right)$ be optimal solutions of $(P 6 b)_{R(i, j)}$ and $(D 6 b)_{R(i, j)}$, respectively. Then the fuzzy weak duality membership function is defined as

$$
\mu^{(R(i, j))}\left(x^{*}, y^{*}\right)=\mu^{P(R(i, j))}\left(x^{*}\right) \wedge \mu^{D(R(i, j))}\left(y^{*}\right) .
$$

Moreover, $\lambda^{t} C x \leq \gamma^{t} D^{t} y$.

To sum up, the approach of Zhong and Shi [93], offers a promising idea about the concept of duality in fuzzy $M C^{2}$ problems, based on the approach of Rödder and Zimmermann [63]. However, the researchers do not prove any other duality results than weak duality.

${ }^{10}$ Here $R(i, j)$ is a pair of ranges for $\gamma$ and $\lambda$. 
Table 2 Literature findings on duality in FLP.

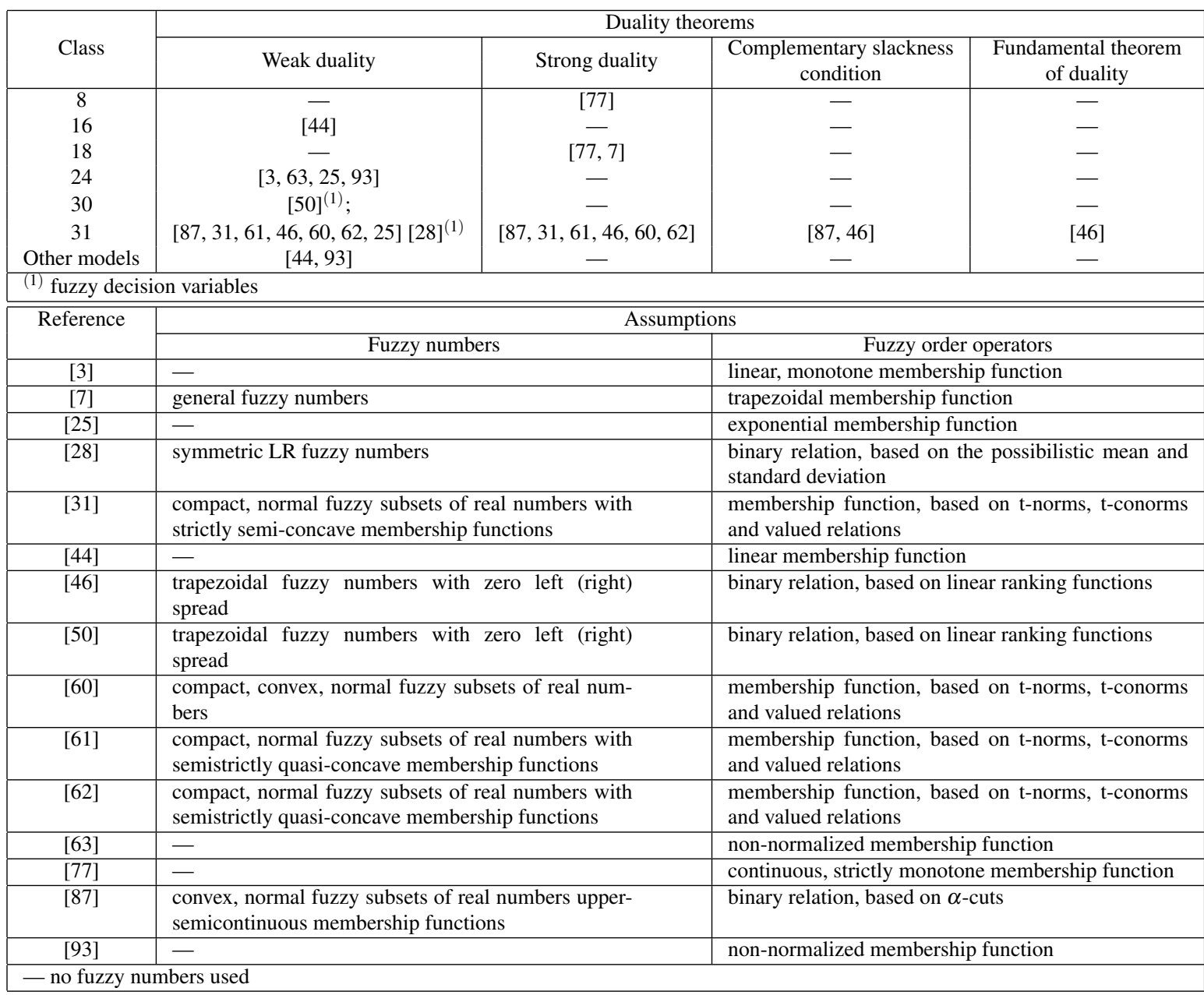

\section{Discussion}

In this section, we present a summary of our findings, identify research gaps, and show avenues for further research. Table 2 summarizes our findings on duality in FLP. The upper part of the table shows which duality theorems have been proven in the literature for which of the classes as defined in Table 1. The lower part shows the mathematical assumptions on fuzzy numbers and fuzzy order operators.

The literature findings shown in Tables 1 and 2 invite for a discussion from two perspectives. The first perspective is class-specific and addresses the question of which duality theorems have been proven for which FLP class. The second perspective is related to the used fuzzy operators and addresses the question of why and how 
literature results show a fragmented picture of FLP duality (theorems). We address both issues in separate subsections before we suggest avenues for further research.

\subsection{Class-specific duality theorems}

We identified 19 relevant papers in the field of FLP. Among these papers, 15 papers contribute to fuzzy duality theory in terms of key duality theorems (weak duality, strong duality, complementary slackness condition, fundamental theorem of duality). The contributions cover only six of 14 potential classes. However, if we regard crisp theorems as specializations of fuzzy theorems, e.g., a crisp number can be regarded as a special fuzzy number, then some classes cover others in the sense that results on fuzzy duality in a more general class can also be applied to more special classes. In more detail, we identify the following coverage relationships:

- Class 24 covers classes 8 and 16. As a consequence, weak duality is shown also for classes 8 and 16 .

- Class 30 covers classes 8, 12, 16, 18, 24, 26 and 28. However, fuzzy numbers need to be trapezoidal numbers with left (right) being zero. Moreover, fuzzy order is defined using ranking functions. Finally, decision variables of models in this class are fuzzy, which limits both theoretical and practical applicability.

- Class 31 covers all other feasible classes. However, theorems are proven by $\mathrm{Wu}$ [87] under very restrictive mathematical conditions and the fuzzy comparison operator is a binary relation. Moreover, Mahdavi-Amiri and Nasseri [46] require fuzzy numbers to be trapezoidal (we identified as an additional requirement that the left (right) spread be zero) and again the use of ranking functions.

To sum up, weak and strong duality theorems in various forms (and partly under different mathematical assumptions) are proven for all feasible classes. In contrast, the complementary slackness condition and the fundamental theorem of duality have been proven explicitly for class 31 and implicitly for all classes only under various, very restrictive assumptions.

\subsection{Operator-specific duality theorems}

As stated in Subsection 2.1, the main idea behind crisp duality theory is that variables are transformed in constraints and vice versa (by using a uniquely defined order operator). It therefore seems straightforward to use this principle to define duality in FLP. However, the main difference between FLP and LP is that the order operator of fuzzy numbers is defined differently as illustrated in Subsection 2.2 and Table 2. Since the definition of the maximization and the minimization operator is based on the definition of the order operator, this also leads to several alternatives for the definition of the maximum and the minimum operator. This, in turn, leads to operator-specific objectives of the fuzzy primal problem and often to operator-specific constraints of the fuzzy dual problem. This phenomenon requires interpreting fuzzy duality theorems in the context of the particular order operator used, in contrast to crisp duality theory 
Fig. 2 Taxonomy of fuzzy order operators

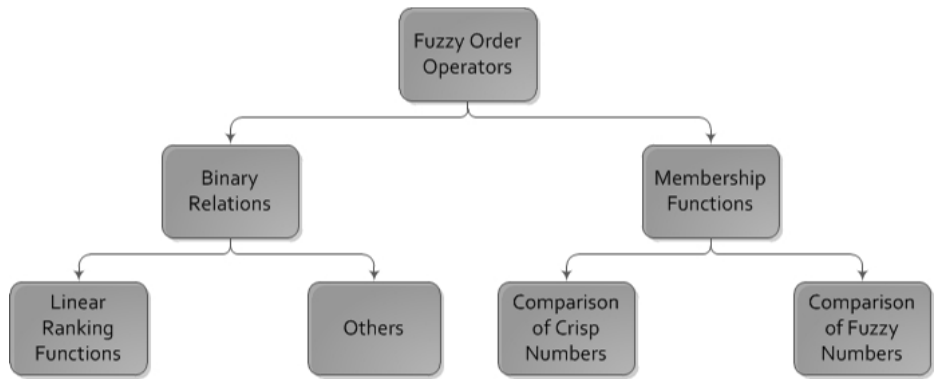

where the order operator, the objective of the primal problem and the constraints of the dual problem are defined uniquely. As a consequence, results on duality in FLP are fragmented.

We now analyze the different order operators with regard to their properties and fuzzy duality theorems proven in terms of homogeneity within the operator group, completeness of duality theorems within the operator group, consistency with crisp duality, and complexity of application. We additionally discuss their advantages and disadvantages. Figure 2 shows four classes of order operators, which can be classified into two main groups: binary relations and membership functions. In the first case, the result of comparing two fuzzy numbers is binary, i.e. either 0 or 1 . In the second case, the result is the degree with which a fuzzy number $\widetilde{A}$ is larger than or equal to a fuzzy number $\widetilde{B}$ or vice versa, i.e. the result is a real number between 0 and 1 . We first consider order operators based on binary relations, which can be further divided into two subgroups: ranking functions [46, 50] and others [87, 28].

Ranking functions transform the decision of comparing two fuzzy numbers into a decision of comparing two crisp numbers. This allows applying the order and the maximum operators in a completely crisp environment, where they are uniquely defined. As a result, the approaches in this group prove (or can easily be extended to prove) all duality theorems consistently to the crisp case. The completeness of the duality theorems, the homogeneous interpretation of duality within the group and the simplicity of application are the main advantages of ranking functions. However, their major disadvantage is that by transforming a decision under uncertainty into one under certainty they fail to reflect important information about the decision situation, which may lead to suboptimal decisions.

The group "Others" of the binary order operators contains all those operators which result in a binary decision but which are not based on ranking functions. As opposed to ranking functions, the order operators in this group incorporate fuzzy uncertainty in the derivation of results. The approach by $\mathrm{Wu}$ [87] compares two fuzzy numbers based on their $\alpha$-cuts while the approach by Hashemi et al. [28] uses possibilistic mean and variance. Both of the approaches prove the weak duality theorem while [87] also proves the strong duality theorem and the complementary slackness theorem. However, the theorems are only partly consistent with crips duality theorems. An advantage of the approaches in this group is that they keep considering 
the uncertainty of the decision environment during the decision process. However, by finally outputting a binary result, this is not mirrored in the result. Moreover, the derivation of duality theorems is not homogeneous within the group and the mathematical setting tends to be complicated, which makes the methods difficult to apply.

The second major group of order operators contains approaches which are based on membership functions. They can be further divided into two subgroups: operators for comparing crisp numbers $[3,77,7,44,63,26,93,25]$ and operators for comparing fuzzy numbers $[31,61,60,62]$.

The operators classified in the first subgroup are developed for the fuzzy comparison of crisp numbers. Naturally, such order operators tend to be relatively simple to apply. The reason for this is that only the uncertainty of the operator has to be reflected but not the uncertainty of numbers. Most of the approaches in this group only prove the weak duality theorem and do not always define duality consistently with the crisp case. In addition, the derivation of duality theorems is generally heterogeneous between the researchers. We identified three subgroups: 1. approaches based on the symmetric principle by Bellman and Zadeh [4]: references [3, 63, 26, 93, 25], 2. approaches that solve a crisp parametric problem [77, 7] and 3. the approach by Liu et al. [44]. We classified the paper by Cadenas Figueredo and Jiménez Barrionuevo [7] in this group because no fuzzy numbers are compared (only maximization of fuzzy objectives) and the solution method is very similar to that used by Verdegay [77]. The main disadvantage of the approaches in this group is that they cannot be generalized for comparing fuzzy numbers so that their applicability is limited.

The second subgroup of order operators based on membership functions are approaches derived from the concepts of t-norms, $t$-conorms and valued relations. They are generally developed for the comparison of fuzzy numbers and thus reflect both the uncertainty of the fuzzy numbers and of the fuzzy order operators. Most of the related papers prove both the weak duality theorem and the strong duality theorem. In addition, they follow a quite homogeneous interpretation of fuzzy duality within the group, which however is generally not consistent with crisp duality. Moreover, they are rather complex to apply.

To sum up, operator groups differ with regard to duality results in terms of the homogeneity of interpretation of fuzzy duality within the group (e.g., high homogeneity in the group "Comparison of fuzzy numbers"), completeness of duality theorems (e.g., in the group "Others": the weak duality theorem, the strong duality theorem and the complementary slackness theorem are proven), consistency with crisp duality (e.g., in the group "Linear Ranking Functions" fuzzy duality is defined analogously to crisp duality) and complexity (e.g., high applicability in the group "Comparison of crisp numbers").

\subsection{Future research}

We see several avenues for further research in FLP duality based on the two perspectives presented in this section. From a class-specific duality properties perspective, we identified the following two research streams: 
1. Research might strive for proving a broader set of duality theorems for selected classes while dispensing with restrictive mathematical assumptions. More precisely, future approaches may concentrate on developing duality theory for FLP classes where only a few components are fuzzified (such as classes 8, 16 and 24) for the sake of making less assumptions in duality theory.

2. Research might look for proving a broader set of duality theorems for general mathematical setting. If, for example, duality theorems are proven for class 31 for general mathematical conditions, researchers would have a broad portfolio of mathematical versions available for all classes. Matching the various sets of formal assumptions with the setting of the specific problems can then lead to the identification and application of appropriate FLP duality results.

From an operator-specific duality properties perspective, we identified the following three research streams:

3. Research might concentrate on further developing duality theory for each of the four operator groups we identified above. In particular, this implies a) striving to achieve a homogeneous definition of duality within the group, b) proving missing duality theorems in the group and c) reducing the complexity of application.

4. Research might strive for developing a unifying fuzzy duality theory. While one could argue in favor of the diversity of requirements and results in FLP duality, we believe that the fragmentation of research in FLP duality makes it difficult to compare the FLP duality research results of different researchers. Furthermore, we argue that the high degree of diversity in FLP duality makes it challenging for those researchers who are interested in drawing on FLP duality results in their specific application context for the purpose of interpreting their results. In the long run, it seems attractive to strive for one unifying FLP duality theory as it is available in crisp linear optimization. The generation of such a theory would leverage the application of FLP duality theory and the relevance of fuzzy linear programming in general.

We believe that a unique definition of the fuzzy order operator would provide the basis for a unifying duality theory. The used order operator should be defined generally enough to be applied to a large number of decision situations (e.g. fuzzy and crisp numbers) and it should adequately reflect the uncertainty the decisionmaker faces (i.e. both in terms of operators and numbers). Moreover, the duality theorems that are developed based on it should have a homogeneous interpretation of duality among researchers and ideally be consistent with crisp duality theory. Therefore, in our opinion, the order operators based on t-norms, t-conorms and valued relations is the best candidate for a unique fuzzy order operator as a basis for the development of a unifying duality theory. Research might thus concentrate on further developing the approaches in this group and deal with the related disadvantages mentioned above.

5. Research in FLP might adapt approaches of duality in fuzzy non-linear programming. Two such examples based on the Lagrangian function are presented in the appendix. 


\section{Concluding Remarks}

In this paper we present a comprehensive overview of the literature results on duality in FLP. We suggest a classification of FLP and use it to structure the presentation of literature findings, including an analysis of the order operators used. We discuss the existing approaches both from a class-specific and operator-specific perspective. Our results show that research on FLP duality is fragmented and incomplete with regard to both FLP classes and order operators. As duality theory (in crisp linear programming) leverages both the mathematical strength and economic relevance of linear programming, we argue that advances in FLP duality would support the diffusion and application of FLP likewise. However, we acknowledge that researchers have already achieved a broad set of results in FLP duality and we suggest paths on how future research can draw upon these in order to strive for more general results in FLP duality. Based on our analysis, we identify the following major future research directions:

(1) Further development of existing methods within their problem class/operator group to facilitate completeness of duality theorems for a particular type of problems,

(2) identification of approaches that are general enough to be applied to a broad set of problem types, and

(3) consideration of alternative approaches, such as those from non-linear optimization, which can be adapted to linear optimization.

While research direction (1) aims at providing a sound duality theory for each group/class, research directions (2) and (3) serve as first steps towards developing a unifying fuzzy duality theory.

We admit that our literature review has some limitations: $(i)$ We have not systematically covered non-linear generalizations of FLP problems. Results in these areas can contribute to duality theory in FLP as the appendix shows. However, covering the literature on fuzzy non-linear optimization would have extended this lengthy literature review substantially. (ii) Due to space limitations, we could only illustrate the key ideas of the approaches but we were not able to explain all of them in detail. Therefore, for some mathematical details, the interested reader needs to consult cited references.

\section{References}

1. Nicolas Abboud, Masahiro Inuiguchi, Masatoshi Sakawa, and Yoshio Uemura. Manpower allocation using genetic annealing. European Journal of Operational Research, 111(2):405-420, December 1998.

2. Adil Baykasoglu and Tolunay Goecken. A review and classification of fuzzy mathematical programs. Journal of Intelligent \& Fuzzy Systems, 19:205-229, August 2008. ISSN 1064-1246.

3. C. R. Bector and S. Chandra. On duality in linear programming under fuzzy environment. Fuzzy Sets and Systems, 125(3):317-325, 2002. ISSN 0165-0114.

4. R. E. Bellman and L. A. Zadeh. Decision-making in a fuzzy environment. Management Science, 17(4):141-164, 1970. 
5. Dimitris Bertsimas and John N. Tsitsiklis. Introduction to Linear Optimization, volume 6 of Athena Scientific Series in Optimization and Neural Computation. Athena Scientific, 1997.

6. Bilge Bilgen. Supply chain network modeling in a golf club industry via fuzzy linear programming approach. Journal of Intelligent \& Fuzzy Systems: Applications in Engineering and Technology, 21:243-253, December 2010. ISSN 1064-1246.

7. J.M. Cadenas Figueredo and F. Jiménez Barrionuevo. A dual approach in fuzzy linear programming. Mathware \& Soft Computing, 3(3), 1996.

8. Christer Carlsson and Robert Fullér. On possibilistic mean value and variance of fuzzy numbers. Fuzzy Sets and Systems, 122(2):315-326, 2001. ISSN 01650114.

9. Stefan Chanas. The use of parametric programming in fuzzy linear programming. Fuzzy Sets and Systems, 11(1-3):229-241, 1983. ISSN 0165-0114.

10. Stefan Chanas and Dorota Kuchta. A concept of the optimal solution of the transportation problem with fuzzy cost coefficients. Fuzzy Sets and Systems, 82 (3):299-305, 1996. ISSN 0165-0114.

11. Stefan Chanas and Dorota Kuchta. Fuzzy integer transportation problem. Fuzzy Sets and Systems, 98(3):291-298, 1998. ISSN 0165-0114.

12. Ni-Bin Chang, C.G. Wen, and Y.L. Chen. A fuzzy multi-objective programming approach for optimal management of the reservoir watershed. European Journal of Operational Research, 99(2):289-302, 1997. ISSN 0377-2217.

13. Cha'o-Kuang Chen and Jin-Mu Lin. A multi-objective fuzzy optimization for optimum dimensions design of a convective spine. International Communications in Heat and Mass Transfer, 28(1):67 - 76, 2001. ISSN 0735-1933.

14. Y.-W. Chen and G.-H. Tzeng. Fuzzy multi-objective approach to the supply chain model. International Journal of Fuzzy Systems, 2:220-228, 2000.

15. Ching-Hsue Cheng. A new approach for ranking fuzzy numbers by distance method. Fuzzy sets and systems, 95(3):307-317, 1998.

16. P. Czyzak. Application of the 'flip' method to farm structure optimization under uncertainty. In R. Slowinski and J. Teghem, editors, Stochastic Versus Fuzzy Approaches to Multiobjective Mathematical Programming under Uncertainty, pages 263-278. Reidei, Dordrecht, 1990.

17. J. Darzentas. On fuzzy location models. In J. Kacprzyk and S.A. Orlovski, editors, Optimization Models Using Fuzzy Sets and Possibility Theory, pages 328-341. Reidei, Dordrecht, 1987.

18. D. Dubois and H. Prade. Operations on fuzzy numbers. International Journal of Systems Science, 9(6):613-626, 1978.

19. D. Dubois and H. Prade. Fuzzy sets and systems: Theory and Applications. Academic Press, New York, 1980.

20. D. Dubois and H. Prade. A review of fuzzy set aggregation connectives. Information Sciences, 36:85-121, 1985.

21. Didier Dubois and Henri Prade. Ranking fuzzy numbers in the setting of possibility theory. Information Sciences, 30(3):183-224, 1983. ISSN 0020-0255.

22. Waiel F. Abd El-Wahed. A multi-objective transportation problem under fuzziness. Fuzzy Sets and Systems, 117(1):27-33, 2001. ISSN 0165-0114. 
23. Geoff Gordon. Linear programming, lagrange multipliers, and duality. http://www.cs.cmu.edu/ ggordon/lp.pdf, n.d.

24. M.M Gupta and E Sanchez. Fuzzy Information and Decision Processes. Elsevier Science Ltd, 1982. ISBN 0444864911.

25. Pankaj Gupta and Mukesh Kumar Mehlawat. Bector-chandra type duality in fuzzy linear programming with exponential membership functions. Fuzzy Sets and Systems, 160:3290-3308, November 2009. ISSN 0165-0114.

26. H. Hamacher, H. Leberling, and H.J. Zimmermann. Sensitivity analysis in fuzzy linear programming. Fuzzy Sets and Systems, 1(4):269-281, 1978.

27. Randolf Hanuscheck. Investitionsplanung auf der Grundlage vager Daten. Wissenschaftliche Schriften, Reihe 2. Schulz-Kirchner, Idstein, 1986. ISBN 3925196196.

28. S. M. Hashemi, M. Modarres, E. Nasrabadi, and M. M. Nasrabadi. Fully fuzzified linear programming, solution and duality. Journal of Intelligent \& Fuzzy Systems, 17(3):253-261, 2006. ISSN 10641246.

29. F.S. Hillier and G.J. Lieberman. Introduction to Operations Research. McGrawHill, 9 edition, 2010.

30. Hsi-Mei Hsu and Wen-Pai Wang. Possibilistic programming in production planning of assemble-to-order environments. Fuzzy Sets and Systems, 119(1):59-70, 2001. ISSN 0165-0114.

31. Masahiro Inuiguchi, Jaroslav Ramík, Tetsuzo Tanino, and Milan Vlach. Satisficing solutions and duality in interval and fuzzy linear programming. Fuzzy Sets and Systems, 135:151-177, April 2003. ISSN 0165-0114.

32. Yakup Kara, Turan Paksoy, and Ching-Ter Chang. Binary fuzzy goal programming approach to single model straight and u-shaped assembly line balancing. European Journal of Operational Research, 195(2):335-347, 2009. ISSN 03772217.

33. E. Karakas, M. Koyuncu, R. Erol, and A. Kokangul. Fuzzy programming for optimal product mix decisions based on expanded abc approach. International Journal of Production Research, 48(3):729-744, 2010.

34. Kuk Kim and Kyung S Park. Ranking fuzzy numbers with index of optimism. Fuzzy sets and Systems, 35(2):143-150, 1990.

35. G.J. Klir and B. Yuan. Fuzzy Sets and Fuzzy Logic: Theory and Applications. Prentice-Hall, New Jersey, 1995.

36. K. K. Lai and Lushu Li. A dynamic approach to multiple-objective resource allocation problem. European Journal of Operational Research, 117(2):293309, 1999. ISSN 0377-2217.

37. Y.-J. Lai and C.-L. Hwang. Fuzzy Multiple Objective Decision Making, Methods and Applications, Lecture Notes in Economics and Mathematical Systems. Springer, Berlin Heidelberg, 1994.

38. Y.-J. Lai and Ch.-L. Hwang. Fuzzy Mathematical Programming: Methods and Applications. Springer-Verlag, 1992.

39. Bum-il Lee, Nam-Kee Chung, and Dong-Wan Tcha. A parallel algorithm and duality for a fuzzy multiobjective linear fractional programming problem. Computers \& Industrial Engineering, 20:367-372, July 1991. 
40. T. León, V. Liern, and E. Vercher. Viability of infeasible portfolio selection problems: A fuzzy approach. European Journal of Operational Research, 139 (1):178-189, 2002. ISSN 0377-2217.

41. Y Leung. Interregional equilibrium and fuzzy linear programming: 2. Environment and Planning A, 20(2):219-230, 1988.

42. Y.P. Li, G.H. Huang, p. Guo, and S.L Nie. Interval-fuzzy possibilistic mixed integer linear programming for environmental management under uncertainty. International Journal of Environment and Pollution, 42(1/2/3):107-124, 2010.

43. Shiang-Tai Liu and Chiang Kao. Solving fuzzy transportation problems based on extension principle. European Journal of Operational Research, 153(3):661674, 2004. ISSN 0377-2217.

44. Y.J. Liu, Y. Shi, and Y.H. Liu. Duality of fuzzy mc2 linear programming: A constructive approach. Journal of Mathematical Analysis and Applications, 194 (2):389-413, 1995. ISSN 0022-247X.

45. N. Mahdavi-Amiri and S.H. Nasseri. Duality in fuzzy variable linear programming. In Proceedings of WEC' 05, The Fourth World Enformatika Conference, volume 6, pages 115-117, Istanbul, Turkey, June 2005.

46. N. Mahdavi-Amiri and S.H. Nasseri. Duality in fuzzy number linear programming by use of a certain linear ranking function. Applied Mathematics and Computation, 180(1):206-216, 2006.

47. N. Mahdavi-Amiri and S.H. Nasseri. Duality results and a dual simplex method for linear programming problems with trapezoidal fuzzy variables. Fuzzy Sets Systems, 158:1961-1978, September 2007. ISSN 0165-0114.

48. K M Mjelde. Fuzzy resource allocation. Fuzzy Sets and Systems, 19:239-250, July 1986. ISSN 0165-0114.

49. S. Mondal and M. Maiti. Multi-item fuzzy EOQ models using genetic algorithm. Computers \& Industrial Engineering, 44(1):105-117, 2003. ISSN 0360-8352.

50. S.H. Nasseri and N. Mahdavi-Amiri. Some duality results on linear programming problems with symmetric fuzzy numbers. Fuzzy Information and Engineering, 1(1):59-66, March 2009.

51. S.H. Nasseri, A. Ebrahimnejad, and S. Mizuno. Duality in fuzzy linear programming with symmetric trapezoidal numbers. Applications and Applied Mathematics: An International Journal (AAM), 5(10):1467-1482, 2010.

52. C. Oder and O. Rentz. Entwicklung eines auf der theorie unscharfer mengen basierenden energie-emissions-modells. In Operations Research Proceedings 1992, pages 111-118, Berin, 1993. Springer-Verlag.

53. Ralf Östermark. Profit apportionment in concerns with mutual ownership - an application of fuzzy inequalities. Fuzzy Sets and Systems, 26(3):283-297, 1988. ISSN 0165-0114.

54. Ralf Östermark. Fuzzy linear constraints in the capital asset pricing model. Fuzzy Sets and Systems, 30(2):93-102, 1989. ISSN 0165-0114.

55. J.W. Owsinski, S. Zadrozny, and J. Kacprzyk. Analysis of water use and needs in agriculture through a fuzzy programming model. In J. Kacprzyk and S.A. Orlovski, editors, Optimization Models Using Fuzzy Sets and Possibility Theory, pages 377-395. Reidei, Dordrecht, 1987. 
56. Ugur Özcan and Bilal Toklu. Multiple-criteria decision-making in two-sided assembly line balancing: A goal programming and a fuzzy goal programming models. Computers \& Operations Research, 36(6):1955-1965, 2009. ISSN 0305-0548.

57. David Peidro, Josefa Mula, Mariano Jimnez, and Ma del Mar Botella. A fuzzy linear programming based approach for tactical supply chain planning in an uncertainty environment. European Journal of Operational Research, 205(1):6580, 2010. ISSN 0377-2217.

58. David Peidro, Josefa Mula, and RaL Poler. Fuzzy linear programming for supply chain planning under uncertainty. International Journal of Information Technology \& Decision Making, 9(03):373-392, 2010.

59. J. Ramík and J. Rimanek. Fuzzy parameters in optimal allocation of resources. In J. Kacprzyk and S.A. Orlovski, editors, Soft Optimization Models Using Fuzzy Sets and Possibility Theory, pages 359-374. Reidei, Dordrecht, 1987.

60. Jaroslav Ramík. Soft computing: Overview and recent developments in fuzzy optimization. http://citeseerx.ist.psu.edu/viewdoc/download? do $i=10.1 .1 .94 .5211 \& r e p=r e p 1 \&$ type=pdf, 2001.

61. Jaroslav Ramík. Duality in fuzzy linear programming: Some new concepts and results. Fuzzy Optimization and Decision Making, 4(1):25-39, February 2005.

62. Jaroslav Ramík. Duality in fuzzy linear programming with possibility and necessity relations. Fuzzy Sets and Systems, 157:1283-1302, May 2006. ISSN 0165-0114.

63. W. Rödder and H.-J. Zimmermann. Duality in fuzzy linear programming. In A.V. Fiacco and K.O. Kortanek, editors, Extremal Methods and System Analysis, pages 415-429. Springer, Berlin, New York, 1980.

64. Heinrich Rommelfanger. Fuzzy linear programming and applications. European Journal of Operational Research, 92(3):512-527, 1996. ISSN 0377-2217.

65. T. K. Roy and M. Maiti. Multi-objective inventory models of deteriorating items with some constraints in a fuzzy environment. Computers \& Operations Research, 25:1085-1095, December 1998. ISSN 0305-0548.

66. T.K. Roy and M. Maiti. A fuzzy eoq model with demand-dependent unit cost under limited storage capacity. European Journal of Operational Research, 99 (2):425-432, 1997. ISSN 0377-2217.

67. M. Sakawa and H. Yano. A fuzzy dual decomposition method for large-scale multiobjective nonlinear programming problems. Fuzzy sets and Systems, 67(1): 19-27, 1994.

68. Masatoshi Sakawa, Ichiro Nishizaki, and Yoshio Uemura. Fuzzy programming and profit and cost allocation for a production and transportation problem. European Journal of Operational Research, 131(1):1-15, 2001. ISSN 0377-2217.

69. Li-Hsing Shih. Cement transportation planning via fuzzy linear programming. International Journal of Production Economics, 58(3):277-287, 1999. ISSN 0925-5273.

70. R. Slowinski. An interactive method for multiobjective linear programming with fuzzy parameters and its application to water supply planning. In Optimization models using fuzzy sets and possibility theory, pages 396-414. Reide, Dordrecht, 1987. 
71. Roman Slowinski. A multicriteria fuzzy linear programming method for water supply system development planning. Fuzzy Sets and Systems, 19(3):217-237, 1986. ISSN 0165-0114.

72. G. Sommer and M.A Pollatschek. A fuzzy programming approach to an air pollution regulation problem. In G.J. Klir R. Trappl and F.R. Pichler, editors, Progress in Cybernetics and Systems Research: General System Methodology, Mathematical System Theory, and Fuzzy Systems, Biocybernetics and Theoretical Neurobiology, volume 3, pages 303-313. Hemisphere, Washington, DC, 1978.

73. T. Spengler. Fuzzy-Entscheidungsmodelle für die Planung der Personalbereitstellung. In W. et. al. Gaul, editor, Operations Research Proceedings 1991, pages 501-508, Berlin, 1992. Springer.

74. Gao-Ji Sun, Yan-Kui Liu, and Yan-Fei Lan. Optimizing material procurement planning problem by two-stage fuzzy programming. Computers \& Industrial Engineering, 58(1):97-107, 2010. ISSN 0360-8352.

75. J. Tang, D. Wang, and R. Y. K. Fung. Fuzzy formulation for multi-product aggregate production planning. Production Planning and Control, 11(7):670-676, 2000.

76. J.F.C. Trappey, C.R. Liu, and T.C. Chang. Fuzzy non-linear programming: Theory and application in manufacturing. International Journal of Production Research, 26:957-985, 1988.

77. J.L. Verdegay. A dual approach to solve the fuzzy linear programming problem. Fuzzy Sets and Systems, 14(2):131-141, 1984. ISSN 0165-0114.

78. J.L. Verdegay. Applications of fuzzy optimization in operational research. Control and Cybernetics, 13:229-239, 1984.

79. Rakesh Verma, M.P. Biswal, and A. Biswas. Fuzzy programming technique to solve multi-objective transportation problems with some non-linear membership functions. Fuzzy Sets and Systems, 91(1):37-43, 1997. ISSN 0165-0114.

80. M. Wagenknecht and K. Hartmann. Fuzzy evaluation of pareto points and its application to hydrocracking processes. In J. Kacprzyk and S.A. Orlovski, editors, Optimization Models Using Fuzzy Sets and Possibility Theory, pages 415-431. Reidei, 1987.

81. P. Z. Wang, Ralf Östermark, Rajan Alex, and S. H. Tan. Using fuzzy bases to resolve nonlinear programming problems. Fuzzy Sets and Systems, 117(1):81 93, 2001. ISSN 0165-0114.

82. Reay-Chen Wang and Tien-Fu Liang. Application of fuzzy multi-objective linear programming to aggregate production planning. Computers \& Industrial Engineering, 46:17-41, March 2004. ISSN 0360-8352.

83. G. Wiedey and H.-J. Zimmermann. Media selection and fuzzy linear programming. The Journal of the Operational Research Society, 29(11):pp. 1071-1084, 1978. ISSN 01605682.

84. Jochen Wolf. Lineare Fuzzy-Modelle zur Unterstützung der Investitionsentscheidung. P. Lang, 1988.

85. Desheng Dash Wu, Yidong Zhang, Dexiang Wu, and David L. Olson. Fuzzy multi-objective programming for supplier selection and risk modeling: A possibility approach. European Journal of Operational Research, 200(3):774-787, 
2010. ISSN 0377-2217.

86. H.-C. Wu. Duality theory in fuzzy mathematical programming problems based on the concepts of necessity. Fuzzy Sets and Systems, 139(139):363-377, 2003.

87. H.-C. Wu. Duality theory in fuzzy linear programming problems with fuzzy coefficients. Fuzzy Optimization and Decision Making, 2(1):61-73, 2003.

88. Hsien-Chung Wu. Duality theory in fuzzy optimization problems. Fuzzy Optimization and Decision Making, 3(4):345-365, December 2004.

89. L. A. Zadeh. The concept of a linguistic variable and its application to approximate reasoning, i. Information Sciences, 8:199-251, 1975.

90. L. A. Zadeh. The concept of a linguistic variable and its application to approximate reasoning, ii. Information Sciences, 8:301-357, 1975.

91. L. A. Zadeh. The concept of a linguistic variable and its application to approximate reasoning, iii. Information Sciences, 9:43-80, 1976.

92. Lotfi Zadeh. Fuzzy sets. Information Control, 8:338-353, 1965.

93. Yihua Zhong and Yong Shi. Duality in fuzzy multi-criteria and multi-constraint level linear programming: a parametric approach. Fuzzy Sets Systems, 132:335346, December 2002. ISSN 0165-0114.

94. H.-J. Zimmermann. Description and optimization of fuzzy systems. International Journal of General Systems, 2(4):209-215, 1976.

95. H. J. Zimmermann. Applications of fuzzy set theory to mathematical programming. Information Sciences, 36(1-2):29-58, 1985. ISSN 0020-0255.

96. H.-J. Zimmermann. Fuzzy sets, decision making, and expert systems. Kluwer, The Netherlands, 1986.

97. H.-J. Zimmermann. Fuzzy set theory-and its applications. Kluwer, Norwell, MA, USA, 4 edition, 2001.

\section{A Literature search}

In our literature search, the following literature databases and journal TOCS (table of contents) were used:

- Literature databases: INFORMS PubsOnLine, databases provided by INFORMS online (Conference Presentation Database, ACI Bibliographic Database), ACM Digital Library, IEEE Xplore, Business Source Premier, MLA International Bibliography, DigiBib of RWTH Aachen University. The logical search string was ("fuzzy" AND "duality").

- Journals: Operations Research, European Journal of Operational Research, Journal on Computing, IIE Transactions, OR Spectrum, INFORMS Journal on Computing, Annals of Operations Research, Mathematical Programming, Fuzzy Sets and Systems, Fuzzy Optimization and Decision Making, Fuzzy Information and Engineering

\section{B Fuzzy non-linear optimization}

\section{B.1 Approach of Sakawa and Yano (1994)}

The papers by Lee et al. [39] and Sakawa and Yano [67] follow a similar idea, with the latter one being more extensive and more generally applicable. We therefore discuss only the second paper in this subsection. Sakawa and Yano [67] consider large-scale multi-objective nonlinear programming problems with fuzzy goals. The primal problem in this paper looks as follows: 


$$
\begin{array}{ll} 
& \widetilde{\min _{x}} f_{1}(x), \ldots, \widetilde{\min _{x}} f_{k}(x) \\
\text { s.t. } & g_{i}(x) \leq 0, i=1, \ldots, m \\
& h_{j}\left(x_{j}\right) \leq 0, j=1, \ldots, p
\end{array}
$$

The researchers pose additional assumptions on the functions $f_{s}, g_{i}, h_{j}$. Note that this is a problem of class 16 in case the objectives and the constraints are linear and there is only one objective. $\widetilde{\text { min }}$ is defined for each of the objective function $f_{s}$ with the help of fuzzy goals. It is specified by the membership function $\mu_{s}$, which is assumed to be invertible on the feasible region for $x$.

To solve $(P b 1)$, the researchers apply the symmetric principle by Bellman and Zadeh [4] and thus rewrite the problem as the following single-objective crisp optimization problem:

$$
\begin{array}{ll}
\min _{x, \alpha}-\alpha \\
\text { s.t. } & f_{s}(x)-\mu_{s}^{-1}(\alpha) \leq 0, s \in 1, \ldots, k \\
& g_{i}(x) \leq 0, i=1, . ., m \\
& x_{j} \in S_{j}, j=1, \ldots, p \\
& \alpha \in[0,1]
\end{array}
$$

Here $S_{j}:=\left\{x_{j} \mid h_{j}\left(x_{j}\right) \geq 0\right\}$ and $S_{j}$ is assumed to be a compact and convex set.

The aim of the researchers is to use a dual decomposition method to cope with the high number of variables in $\left(P b 1^{\prime}\right)$, which makes the problem practically unsolvable for realistic applications. However, to apply the above method, the objective function needs to be strictly convex, which is not the case for $\left(P b 1^{\prime}\right)$. Sakawa and Yano [67] thus approximate the problem $\left(P b 1^{\prime}\right)$ with

$$
\begin{array}{ll}
\min _{x, \alpha} & -\alpha+\rho \alpha^{2} \\
\text { s.t. } & f_{s}(x)-\mu_{s}^{-1}(\alpha) \leq 0, s \in 1, \ldots, k \\
& g_{i}(x) \leq 0, i=1, \ldots, m \\
& x_{j} \in S_{j}, j=1, \ldots, p \\
& \alpha \in[0,1],
\end{array}
$$

where $\rho$ is a very small, positive number.

The Lagrangian of $\left(P b 1^{\prime \prime}\right)$ is defined as

$$
L(x, \alpha, \lambda, \pi)=\sum_{j}\left(\sum_{s} \lambda_{s} f_{s j}\left(x_{j}\right)+\sum_{i} \pi_{i} g_{i j}\left(x_{j}\right)\right)+\left(-\alpha+\rho \alpha^{2}-\sum_{s} \lambda_{s} \mu_{s}^{-1}(\alpha)\right),
$$

where $\lambda$ and $\pi$ are vectors of the corresponding Lagrangian multipliers. Let be

$$
\begin{array}{ll}
w(\lambda, \pi):= & \min _{x, \alpha} L(x, \alpha, \lambda, \pi) \\
\text { s.t. } & x_{j} \in S_{j}, j=1, \ldots, p \\
& \alpha \in[0,1] .
\end{array}
$$

The crisp dual problem of $\left(P b 1^{\prime \prime}\right)$ is then

$$
\begin{aligned}
& \max _{\lambda, \pi} w(\lambda, \pi) \\
& \text { s.t. }(\lambda, \pi) \in U,
\end{aligned}
$$

where $U=:\{\lambda>0, \pi>0: w(\lambda, \pi)$ exists $\}$.

Strong duality The optimal solution of problem $\left(\mathrm{Pb}^{\prime \prime}\right)$ coincides with the optimal solution of problem $(\mathrm{Db} 1)$.

To sum up, the idea of Sakawa and Yano [67] is to solve the computationally complex primal problem using the simpler dual problem. The approach is quite interesting, but it concentrates mainly on solving the dual problem rather than developing a fuzzy duality theory. In addition, the dual problem is not a fuzzy problem anymore. 


\section{B.2 Approach of Wu (2003)}

Wu [86] considers a general fuzzy optimization problem. The primal problem in this paper is defined as

$$
\begin{array}{ll}
\widetilde{\min }_{x} & \widetilde{f(x)} \\
\text { s.t. } & \widetilde{g(x)} \preceq 0 \\
& x \in X .
\end{array}
$$

Here $\widetilde{f}$ and $\widetilde{g}$ are fuzzy-valued functions and $X$ is a convex subset of the real vector space. Note that for the special case where $\widetilde{f(x)}=\widetilde{c^{t}} x$ and $\widetilde{g(x)}=\widetilde{A} x-\widetilde{b}$ the problem belongs to class 31 . The researcher works with convex, normal fuzzy subsets of the real numbers with upper-semicontinuous membership functions and compact $\alpha$-cut for $\alpha=0$.

They define $\preceq$ using necessity indices as defined by Dubois and Prade [21]. Given two fuzzy numbers $\widetilde{a}$ and $\widetilde{b}$ with membership functions $\mu_{\tilde{a}}$ and $\mu_{\tilde{b}}$, the necessity index is defined as

$$
\begin{aligned}
& \text { Nece }(\widetilde{a} \succeq \widetilde{b})=\inf _{u} \sup _{v: u \geq v} \max \left\{1-\mu_{\widetilde{a}}(u), \mu_{\tilde{b}}(v)\right\} \\
& \operatorname{Nece}(\widetilde{a} \succ \widetilde{b})=1-\sup _{u \leq v} \max \left\{\mu_{\widetilde{a}}(u), \mu_{\tilde{b}}(v)\right\} .
\end{aligned}
$$

Then it holds that

$$
\widetilde{a} \succeq_{\alpha} \widetilde{b} \Leftrightarrow \operatorname{Nece}(\widetilde{a} \succeq \widetilde{b}) \geq \alpha \cap N e c e(\widetilde{a} \succ \widetilde{b}) \geq \alpha, \alpha \in[0,1] .
$$

The optimal solution for $(P b 2)$ is the feasible solution where the objective function has the lowest value according to the definition of the fuzzy comparison operator. Note that, due to the particular definition of $\succeq_{\alpha}$, a feasible solution is always defined for a given $\alpha$. Similarly, an optimal solution is also defined for a given $\alpha^{\prime}$, characterizing the comparison operator of the objective values, i.e. $\widetilde{\min ^{\alpha^{\prime}}}$. Moreover, $\alpha$ may differ from $\alpha^{\prime}$. Following the researcher, we call such a problem an $\left(\alpha^{\prime}, \alpha\right)-(P b 2)$ problem.

To define the dual problem to $(P b 2)$, the researcher follows the same approach as in crisp nonlinear programming. They define the fuzzy-valued Lagrangian function of an $\left(\alpha^{\prime}, \alpha\right)-(P b 2)$ problem as follows:

$$
\widetilde{\phi(x, u)}=\widetilde{f(x)}+u^{t} \widetilde{g(x)}, x \in X
$$

where $u>0$. The fuzzy-valued Lagrangian dual function for a given $\widetilde{\min ^{\beta}}$ is thus (here $\beta$ may be equal to $\alpha$, but does not have to)

$$
L_{\beta}(u)=\widetilde{\min ^{\beta}} \underset{x \in X}{\phi(x, u)} .
$$

The fuzzy dual problem is thus defined as

$$
\begin{array}{ll}
\widehat{\max \beta^{\prime \prime}}{ }_{u} & L_{\beta}(u) \\
\text { s.t. } & u \geq 0 .
\end{array}
$$

As the problem depends on $\beta$ and $\beta^{\prime}$, we call it a $\left(\beta^{\prime}, \beta\right)-(D b 2)$ problem. Note that $\widetilde{\max } \beta^{\prime}$ requires a definition that is slightly different from that of $\min ^{\beta^{\prime}}$. The interested reader can consult reference [86].

Weak duality (1) Let $x$ and $u$ respectively be feasible solutions of an $\left(\alpha^{\prime}, \alpha\right)-(P b 2)$ and a $\left(\beta^{\prime}, \alpha\right)-(D b 2)$ problem, where $\beta^{\prime}$ may also equal $\alpha^{\prime}$. Then, under some additional assumptions, it holds that

$$
L_{\alpha}(u) \preceq_{\alpha} \widetilde{f(x)}, \alpha \geq 0.5 .
$$

Weak duality (2) Let $x^{*}$ and $u^{*}$ respectively be optimal solutions of an $(\alpha, \alpha)-(P b 2)$ and an $(\alpha, \alpha)-$ $(D b 2)$ problem. Then, under some assumptions, it holds that

$$
L_{\alpha}\left(u^{*}\right) \preceq_{\alpha} \widetilde{f\left(x^{*}\right)}, \alpha \leq 0.5 .
$$

Note that weak duality (2) is a special case of weak duality (1). In addition, the researcher provides conditions for the optimality of two feasible solutions of the primal and the dual problem, respectively. 
Strong duality Under some assumptions, the problems $(\alpha, \alpha)-(P b 2)$ and $(\alpha, \alpha)-(D b 2)$ have no duality gap for $\alpha \geq 0.5$.

To sum up, the main advantage of this approach is that it can be applied to a number of fuzzy optimization problems (satisfying the necessary assumptions) and it applies the ideas of crisp duality theory without defuzzifying the problems. However, the mathematical setup is rather complicated. In a follow-up paper [88], the researcher deals with the above problem by modifying the definition of fuzzy order and explaining the concepts with examples. Since the ideas in this paper are very similar to the one we discussed in this section, we omit its review here. 